\title{
IBEX Backgrounds and Signal-to-Noise Ratio
}

\author{
P. Wurz • S.A. Fuselier · E. Möbius · H.O. Funsten • P.C. Brandt • F. Allegrini • \\ A.G. Ghielmetti · R. Harper · E. Hertzberg • P. Janzen • H. Kucharek • \\ D.J. McComas · E.C. Roelof $\cdot$ L. Saul $\cdot$ J. Scheer $\cdot$ M. Wieser $\cdot$ Y. Zheng
}

Received: 16 January 2009 / Accepted: 21 April 2009 / Published online: 9 June 2009

(C) Springer Science+Business Media B.V. 2009

\begin{abstract}
The Interstellar Boundary Explorer (IBEX) mission will provide maps of energetic neutral atoms (ENAs) originating from the boundary region of our heliosphere. On IBEX there are two sensors, IBEX-Lo and IBEX-Hi, covering the energy ranges from 10 to $2000 \mathrm{eV}$ and from 300 to $6000 \mathrm{eV}$, respectively. The expected ENA signals at $1 \mathrm{AU}$ are low, therefore both sensors feature large geometric factors. In addition, special attention has to be paid to the various sources of background that may interfere with our measurement. Because IBEX orbits the Earth, ion, electron, and ENA populations of the Earth's magnetosphere are
\end{abstract}

P. Wurz $(\bowtie) \cdot$ L. Saul $\cdot$ J. Scheer

Physics Institute, University of Bern, Sidlerstrasse 5, 3012 Bern, Switzerland

e-mail: peter.wurz@space.unibe.ch

S.A. Fuselier · A.G. Ghielmetti · E. Hertzberg

Space Physics Department, Lockheed Martin Advanced Technology Center, 3251 Hanover Street,

Palo Alto, CA 94304, USA

E. Möbius · H. Kucharek

Space Science Center and Department of Physics, University of New Hampshire, 39 College Road,

Durham, NH 03824, USA

H.O. Funsten · R. Harper · P. Janzen

Los Alamos National Laboratory, Los Alamos, NM 87545, USA

M. Wieser

Swedish Institute of Space Physics, Box 812, 98128 Kiruna, Sweden

P.C. Brandt · E.C. Roelof · Y. Zheng

Applied Physics Laboratory, 11100 Johns Hopkins Road, Laurel, MD 20723, USA

F. Allegrini · D.J. McComas

Space Science and Engineering Division, Southwest Research Institute, San Antonio, TX 78228-0510, USA

Present address:

P. Janzen

Department of Physics \& Astronomy, University of Montana, 32 Campus Drive, Missoula, MT 59812, USA 
prime background sources. Another potential background source is the magnetosheath and the solar wind plasma when the spacecraft is outside the magnetosphere. UV light from the night sky and the geocorona have to be considered as background sources as well. Finally background sources within each of the sensors must be examined.

Keywords Interstellar boundary · Energetic Neutral Atom · ENA · Instrumentation

\section{Introduction}

The Interstellar Boundary Explorer (IBEX) mission will explore in detail the boundaries of our heliosphere, which is where the solar wind interacts with the interstellar medium (see McComas et al. 2009, this issue). For that purpose, IBEX produces full-sky maps of energetic neutral atoms (ENAs) originating from the boundary region of our heliosphere. There are two sensors, IBEX-Lo and IBEX-Hi, described in detail by Fuselier et al. (2009, this issue) and Funsten et al. (2009, this issue), respectively. IBEX-Lo and IBEX-Hi cover the energy ranges from 10 to $2000 \mathrm{eV}$ and from 300 to $6000 \mathrm{eV}$, in eight and six energy steps, respectively.

The ENA signals from the boundaries of the heliosphere are expected to be low (Wurz et al. 2008). Thus both sensors feature large geometric factors. In addition, special attention has to be paid to the various sources of background that may interfere with our measurements. As with most applications of ENA instruments in space the fluxes of unwanted signal sources are much larger than the ENA flux, and their efficient suppression is crucial for successful ENA measurements (Wurz 2000). IBEX is in a highly elliptical Earth orbit of about $3 \times 50 R_{E}$ (Earth radii). Thus, ion and electron populations of the Earth's magnetosphere and in the solar wind when the spacecraft is outside the magnetosphere are prime sources for background particles. Moreover, ENAs emitted from the terrestrial magnetosphere are of much higher intensity than the expected interstellar signal. Additional background sources include cosmic rays and diffuse UV light backscattered from the interstellar gas and geocorona. Finally, there are also some internal background sources typical for each sensor that need careful consideration.

\subsection{Expected Signal}

Because of the obvious observational difficulties and the lack of dedicated missions, there are, at present, only a few measurements available on ENAs from beyond the termination shock region. The existing ENA measurements from the termination shock region have been summarised in Fig. 1. They range from $200 \mathrm{eV}$ upwards to almost $100 \mathrm{keV}$ (Hilchenbach et al. 1998; Kallenbach et al. 2005; Wurz et al. 2008). When integrated over energy the ENA fluxes are found to be low with typical values of the order of $10^{4} \mathrm{~cm}^{-2} \mathrm{~s}^{-1} \mathrm{sr}^{-1}$ (Wurz et al. 2008). Most recently, Wang et al. (2008) reported such ENA measurements above a few keV from the STEREO mission. However, these measurements have later been reinterpreted to be X-rays from stars near the galactic centre (Hsieh et al. 2009).

At present, there are no ENA measurements reported below $200 \mathrm{eV}$. Wood et al. (2007) extrapolated the ENA measurements to lower energies by comparing them with Lyman- $\alpha$ absorption measurements. Based on these extrapolations it was concluded that a straight continuation of the ENA spectrum to lower energies is in conflict with the profiles of the Lyman- $\alpha$ absorption. At energies at and below about $100 \mathrm{eV}$ some sort of flattening of the slope or even a roll over is expected, as suggested by several model calculations (see also Fig. 1). 


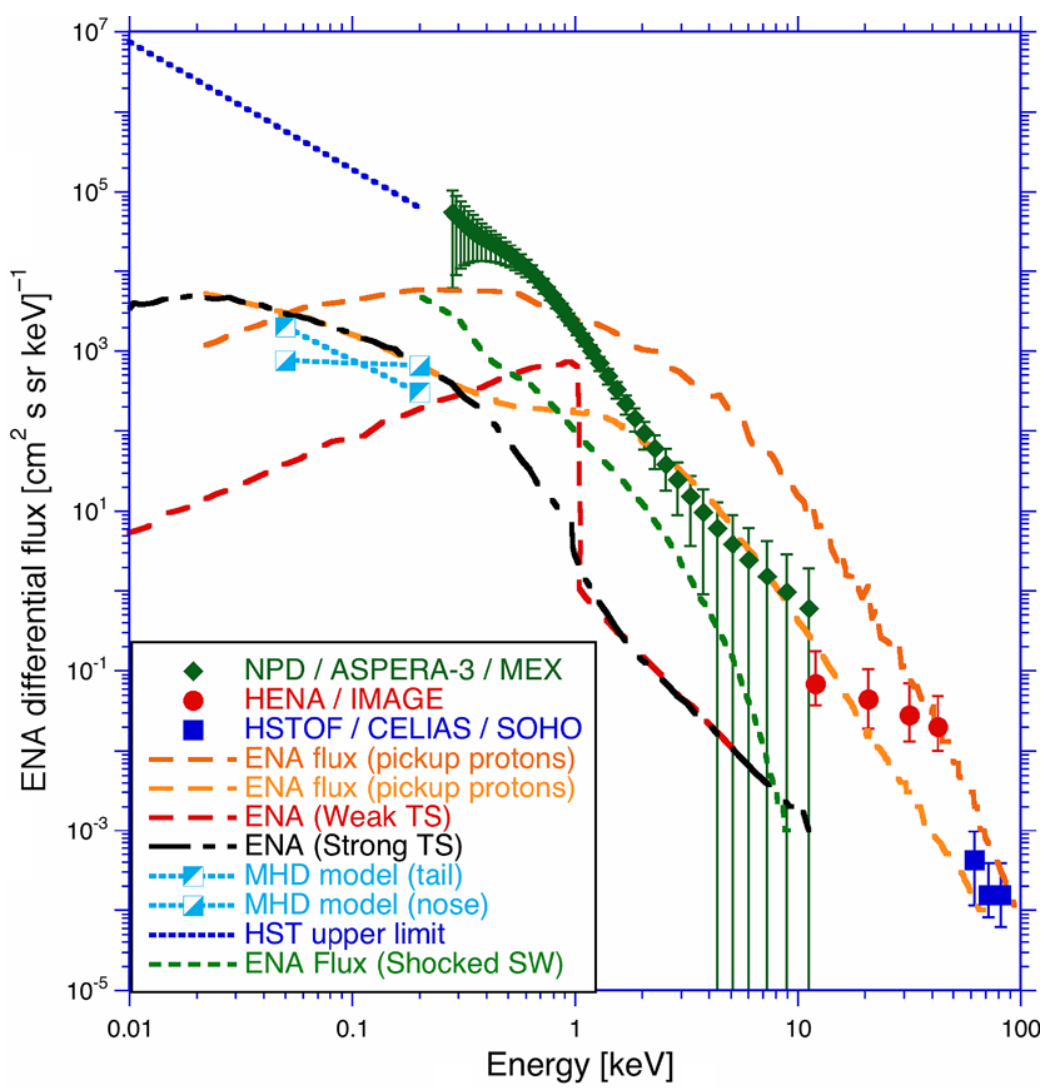

Fig. 1 Points with error bars: Compilation of experimental data of ENAs from the termination shock region. The red circles are not actual ENAs from the termination shock region, but represent an upper limit to these fluxes. The lines give model fluxes. For details see main text

Our present understanding of the global properties of the region beyond the termination shock relies mostly on detailed model calculations. These calculations not only predict the shape and the physical parameters in the various regimes of the heliospheric interface, but they also predict energy spectra of ENAs produced in that region and travelling inward to Earth orbit. Several calculated ENA energy spectra for an observer at Earth orbit are reproduced in Fig. 1 as well (Wurz et al. 2008, and references therein). The black dashed and red dashed-dotted lines in Fig. 1 (McComas et al. 2004) represent a strong and a weak termination shock, respectively. The light blue lines represent MHD model nose and tail data from a self-consistent axisymmetric MHD plasma/kinetic neutral model (Heerikhuisen et al. 2007). The green short-dashed line shows ENA flux for shocked solar wind data from Fahr and Scherer (2004); the orange long-dashed curves represent two cases of ENA flux for pickup protons from the calculation by Chalov et al. (2003). The HST measurements are indicated by the dotted line that represents an upper limit for the ENA fluxes derived from the observations by Wood et al. (2007).

Currently, there is still is a large range in the predictions of ENA intensities by the various models as can be seen in Fig. 1. For our discussions of the background levels we will use the ENA predictions for the weak and strong termination shocks shown in Fig. 1, which are 
from the model by Gruntman et al. (2001), since they represent conservative assumptions for the evaluation of the signal-to-noise ratio.

\subsection{Background and Suppression Requirements}

Based on careful analysis of the expected ENA signal from the termination shock region and the corresponding count rates in the sensors, requirements were developed for the suppression of background and of unwanted stimuli and noise. These requirements were developed to make sure that resulting background and noise count rates will be well below the expected signal rates. Thus requirements for the suppression were defined based on the evaluation of the charged particle and UV environment at the location of the IBEX spacecraft. These requirements are listed below.

\subsubsection{IBEX-Lo Requirements}

- Noise (excluding cosmic rays) for IBEX-Lo shall be $\leq 8 \cdot 10^{-3}$ counts/sec for all acceptable "any double" coincidence events. Acceptable means that the measured time-of-flight is consistent with the detection of an ion.

- The goal for noise (excluding cosmic rays) for IBEX-Lo is $\leq 8 \cdot 10^{-4}$ counts/sec for all acceptable "any double" coincidence rate.

- Average background for IBEX-Lo shall be $<2 \cdot 10^{-1} \mathrm{ENA} / \mathrm{sec} /$ time pixel in energy range between $200 \mathrm{eV}$ and $1 \mathrm{keV}$ incident at the entrance to the energy analysis subsystem.

- The goal for average background is $<2 \cdot 10^{-2} \mathrm{ENA} / \mathrm{sec} / \mathrm{time}$ pixel in energy range between $200 \mathrm{eV}$ and $1 \mathrm{keV}$ incident at the entrance to the energy analysis subsystem.

- Signal-to-noise ratio for IBEX-Lo shall be $>1$.

- The total singles rate from photons and electrons should be $<1000 / \mathrm{sec}$.

- Maximum illegitimate triple coincidence count rate (all other particles masquerading as signal) should be 10 per day when IBEX is in the solar wind.

- The IBEX-Lo collimator shall reject ions of energy $\leq 10 \mathrm{keV}$. The reduction in integral flux shall be $>100$ (goal $>1 \cdot 10^{4}$ ) for energies $<10 \mathrm{keV}$.

- The IBEX-Lo collimator shall reject electrons of energy less than $600 \mathrm{eV}$. The reduction in integral flux shall be $>1 \cdot 10^{4}$ for energies $<150 \mathrm{eV}$, and $>100$ for energies between 150 and $600 \mathrm{eV}$.

- IBEX-Lo shall deflect and separate the ionised ENAs from ambient UV light and ions of energy greater than $10 \mathrm{keV}$.

- IBEX-Lo shall use low outgassing materials with strict control of organic volatiles.

- The IBEX-Lo TOF electronics shall not vent through or into the optical or TOF detector subsystems.

- The IBEX-Lo optics and TOF subsystems shall not vent through or into the interior of the spacecraft.

\subsubsection{IBEX-Hi Requirements}

- The electrostatic energy analyser shall incorporate anti-reflection measures including serrations and two-bounce pass minimum for background particles to enter detector subsystem.

- IBEX-Hi shall have a coincidence subsystem that provides coincidence detection of ionised ENAs that transit the energy analysis subsystem.

- The IBEX-Hi individual channel electron multiplier (CEM) background count rate shall be less than 1 count/sec. 
- The IBEX-Hi triple coincidence count rate of the detector subsystem in the solar wind shall be less than $2 \cdot 10^{-4}$ counts/sec. This corresponds to a background flux of atoms or ions at the conversion foil of $3.8 \cdot 10^{-2}$ particles $/ \mathrm{sec}$.

- The intrinsic IBEX-Hi triple coincidence count rate of the detector subsystem in the solar wind should be less than $1 \cdot 10^{-5}$ counts/sec.

- The IBEX-Hi detector subsystem shall reside in a light-tight enclosure having minimum 2-corner flange pockets for all edges of enclosure parts.

- IBEX-Hi shall use low outgassing materials with strict control of organic volatiles per the IBEX Contamination Control Plan.

- The IBEX-Hi detector electronics shall not vent through the collimator subsystem.

- The IBEX-Hi ESA and detector subsystems shall not vent through or into the interior of the spacecraft.

- In the solar wind IBEX-Hi shall have a background count level $<2 \cdot 10^{-2}$ ENAs/sec at the entrance to the energy analysis subsystem in the energy ranges from $1 \mathrm{keV}$ to $3 \mathrm{keV}$.

- The above background rate should be less than $2 \cdot 10^{-4}$ counts/sec in the energy ranges from $1 \mathrm{keV}$ to $3 \mathrm{keV}$ and at the entrance to the energy analysis subsystem in the solar wind.

\subsection{Principles of Background Suppression}

Because the expected signal levels of ENAs arriving from the termination shock region are extremely low compared with other diffuse particle and photon sources, any sources of background must be efficiently suppressed to enable a sufficient signal-to-noise ratio to answer the four fundamental science questions underlying the IBEX mission. Major sources of background include charged particles in the local space environment, i.e., electrons and ions, as well as UV photons and penetrating energetic particles. To cope with such demanding requirements background suppression was a key driver for the subsystems of each sensor. In this first section, we start with a brief overview of the general measurement techniques used by both sensors. Section 2 follows with a quantitative evaluation of all identified external background sources. Section 3 will then describe background sources specific to the sensor designs and how they have been minimised.

Both sensors employ the same basic principles to image and detect ENAs and suppress background. They use a succession of mechanical collimation for imaging, conversion of neutrals into ions for further analysis, electrostatic collection and energy analysis, postacceleration, and coincidence detector techniques (double and triple) for unambiguous signal identification and to achieve the lowest possible background levels. IBEX-Lo converts ENAs to negative ions, which is most efficient at low energies (Wurz 2000), and IBEX-Hi converts them into positive ions, which is most efficient at higher energies. This difference leads to background considerations that are specific to each sensor. Therefore, we start with a brief compilation of background suppression in each of the four subsystems, followed by some notes on specific features in IBEX-Lo and IBEX-Hi.

\subsubsection{Common Background Suppression Concepts}

Charged Particle Suppression: As will be shown in more detail below, charged particles are many orders of magnitude more abundant than ENAs and are electrostatically rejected at the aperture, before even reaching any sensor structure. This rejection is implemented with negatively biased annular electrodes in front of the collimator aperture but outside of the sensor field-of-view (FOV) to repel ambient electrons with energies up to $600 \mathrm{eV}$. Electron 
rejection is followed by electrostatic rejection of positive ions by applying a $+10 \mathrm{kV}$ bias directly to the collimator stack, resulting in rejection of ions with energies up to $10 \mathrm{keV}$. Because the charged particle suppression introduces secondary background sources within the collimator that need to be understood when discussing the external background environment, the charged particle rejection is described in more detail at the end of this section.

Electrostatic Analyser (ESA): After conversion of the ENAs into ions (negative ions for IBEX-Lo and positive ions for IBEX-Hi), the ESA selects the energy passbands for observation, and ions with energies outside of the passband are prevented from transiting the ESA and entering the detector subsystem. Ions outside the selected energy passband will impact an ESA plate and be absorbed in the structure of the ESA. The curvature of the ESA plates likewise suppresses the propagation of UV photons, which impact the outer ESA plate first. Furthermore, the ESA design requires a minimum two-bounce path for any scattered photon to enter the detector subsystem, further suppressing the small fraction of photons that reflect within the ESA subsystem. For IBEX-Lo, the suppression is increased through the use of copper sulphide blackening of all surfaces and serrations of the outer ESA electrode. For IBEX-Hi, the suppression is enhanced by serrations and a dendritic Ebonol-C coating.

Coincidence Measurements: For the successful identification of an ion, at least two detections (at a start and a stop detector) are required in both sensors. A coincident detection event is considered valid if these sequential detections lie within a prescribed time window. The coincidence technique substantially reduces background from random events, such as those arising from penetrating radiation, UV, or detector dark counts. To reduce the background even further, a triple coincidence measurement has been implemented that requires three detections each in a different detector with a specific temporal sequence. Triple coincidence events have the largest background suppression and thus comprise the highest signal-to-noise measurement of ENAs.

Zero Energy Bin: In addition to the eight ENA passbands for IBEX-Lo and six energy passbands IBEX-Hi, there is a zero energy bin where the ESA voltages are set near zero, which prevents ions from passing through the ESA toward the detector subsystem. For this ESA voltage setting, the coincident and non-coincident count rates from UV, cosmic ray, and internal background sources can be measured in orbit for each sensor. This zero energy bin will be used periodically throughout the mission to assess background levels.

Background Detector: To assess the actual energetic ion environment of IBEX a background ion detector was added to IBEX-Hi that measures the integral flux of ions with energies above $14 \mathrm{keV}$ (see Allegrini et al. 2009, this issue).

\subsubsection{Specific IBEX-Lo Background Suppression Concepts}

IBEX-Lo works at the low end of the ENA energy range and, after ENAs traverse the collimator, converts them to negative ions. These characteristics provide two additional challenges. Ultraviolet light that enters the collimator unimpeded and strikes either collimator structure or the conversion surface can generate copious photo-electrons. Electrons (i.e., photo-electrons and secondary electrons) released from the conversion surface are accelerated towards the ESA. Because of their negative charge, electrons can pass through the ESA provided their energy is inside the selected ESA energy passband. Therefore, magnets were implemented as an additional suppression stage that is not needed in IBEX-Hi. 
Magnets: Between the conversion surface unit and the entrance to the ESA there are two nearly concentric rings of permanent magnets that suppress the passage of secondary electrons (from UV or particle induced) into the ESA (see Wieser et al. 2007; Fuselier et al. 2009, this issue).

Time-of-Flight (TOF) Measurement: IBEX-Lo is also required to distinguish different species, such as $\mathrm{H}$ and $\mathrm{O}$. Therefore, the detector system is a TOF mass spectrometer (see Fuselier et al. 2009, this issue). With the triple coincidence measurement IBEX-Lo even provides three TOF measurements, which need to be consistent for actual particles, thus providing unprecedented background suppression.

\subsubsection{Specific IBEX-Hi Background Suppression Concepts}

In IBEX-Hi, ENAs that pass through the collimator transit an ultrathin charge conversion foil. Ultraviolet can likewise pass through the collimator and generate photo-electrons at the entrance surface of the foil and exposed parts of the foil frame. Because the collimator is nominally biased to $+10 \mathrm{kV}$, these photo-electrons would be accelerated towards the collimator and ionise any residual gas between the foil and the collimator. These ions are accelerated towards the foil at an energy between nearly $0 \mathrm{~V}$ (if ionisation occurs near the foil surface) and $10 \mathrm{keV}$ (if ionisation occurs in the collimator). Ions impacting the foil at energies $\lesssim 7 \mathrm{keV}$ can masquerade as heliospheric ENAs. To mitigate this ion production by photo-electrons, a high (95\%) transmission grid biased to $-300 \mathrm{~V}$ was placed immediately above the foil to suppress photo-electrons from the foil and foil frame by a factor of 20 .

Coincidence System: IBEX-Hi employs a simpler coincidence detection system than IBEX-Lo because mass analysis of the ENAs is not necessary. To provide superior background suppression, specific coincidence timing windows have been implemented. Different combinations of such windows provide a range of signal-to-noise values (Funsten et al. 2009, this issue).

\subsubsection{The Collimator Subsystem}

The collimator system plays a crucial role in the suppression of incoming charged particles that would interfere with the performance of the two sensors. The collimator subsystems for IBEX-Lo and IBEX-Hi are very similar, and the subsystem performance is explained in detail in the two instrument papers (Fuselier et al. 2009, this issue; Funsten et al. 2009, this issue). Here we will only address the suppression of unwanted particle fluxes and possible background signals that have their origin in the collimator system. The necessary sequence of electrodes at negative and positive bias potentials has to be considered in detail in the analysis.

Figure 2 shows a schematic diagram of the IBEX collimator (top panel) and the voltages applied to the grids (bottom panel). In the entrance aperture, a negative high voltage, $-\mathrm{HV}$, is applied to reject external electrons with energies up to $600 \mathrm{eV}$. The mechanical collimator itself is at a high positive voltage, $+\mathrm{HV}$, to reject ions in the local space environment with energies up to $10 \mathrm{keV} / \mathrm{q}$. Charged particles with higher energies can pass through the collimator into the charge conversion subsystem, but their transmission is largely reduced by the geometrical collimation of the hexagonal channels and the electrostatic defocusing property of the pre-collimator. To assist in understanding the background from ions with energy larger than $10 \mathrm{keV}$, the IBEX Background Monitor (IBaM, Allegrini et al. 2009, this 


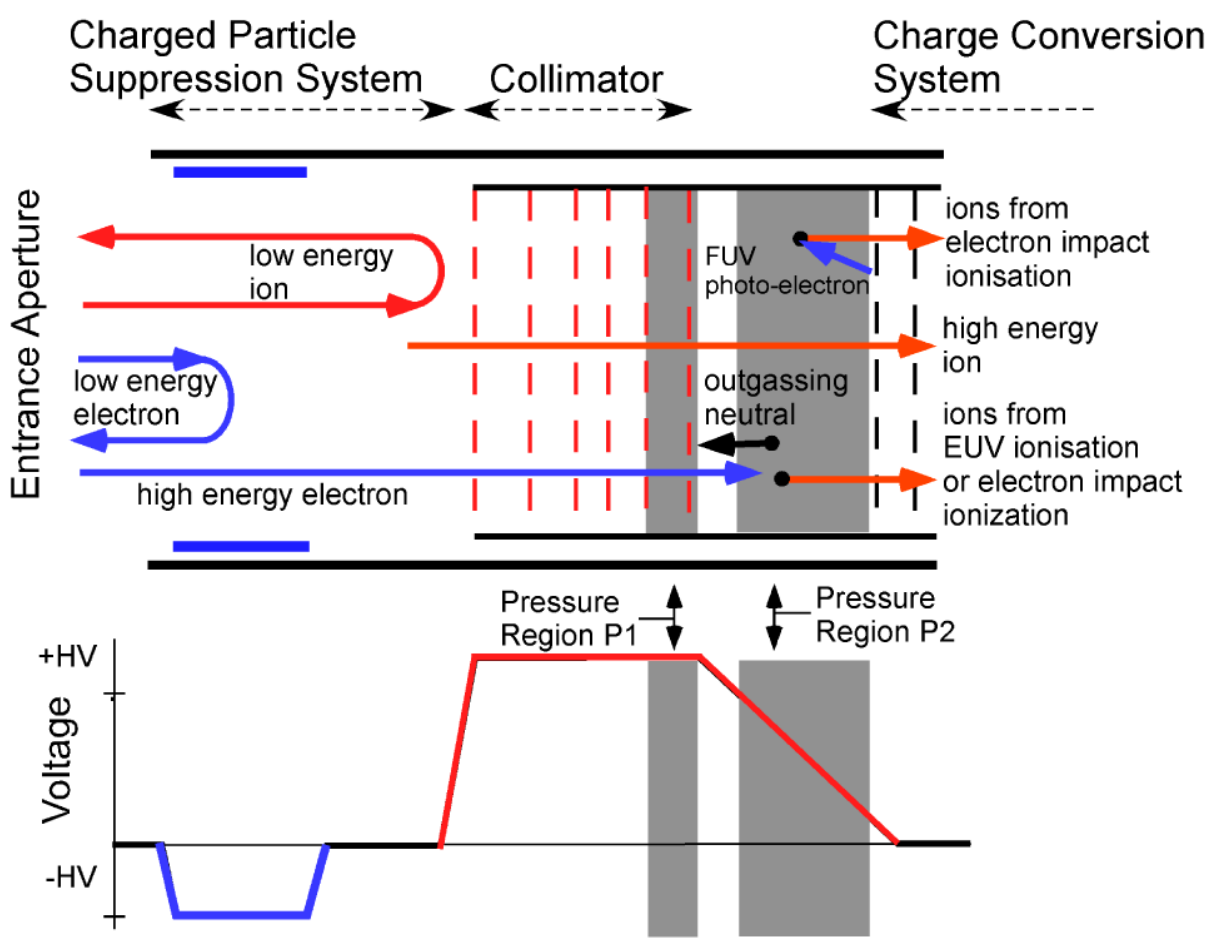

\section{Distance in Entrance/Collimator}

Fig. 2 Schematic of the IBEX collimator (top) and voltages applied to the grids (bottom). Source neutrals, ions, and electrons enter from the entrance aperture on the left hand side

issue), which is incorporated within and boresighted with the IBEX-Hi sensor, will measure the integral fluxes of ions in the local space environment with energies $\gtrsim 14 \mathrm{keV}$ within a FOV identical to IBEX-Hi and IBEX-Lo.

Even after employing the most careful cleanliness procedures in the sensor design, sensor components will continuously outgas, and a residual gas will be present within the collimator system. The partial pressure of the gas species depends on the area of the outgassing components, the outgassing rate of these components, and the conductance through the collimator subsystem. The volume between the last collimator grid and the charge conversion subsystems of both sensors is of particular concern because of the ion production in which secondary electrons, photo-electrons, and UV photons ionise the residual gas atoms and molecules, which are then accelerated into the charge conversion subsystem. The sensitive volume for the generation of background ions that can masquerade in the sensor as legitimate ENAs are the two pressure regions P1, which lies within the collimator as shown in Fig. 2, and P2, which is between the collimator and the charge conversion subsystem. Because the composition of the residual gas in space is expected to be dominated by water vapour we focus the discussion on water vapour in the following analysis.

There are two external sources for the generation of background ions near the exit region of the collimator (pressure region P1 shown schematically in Fig. 2) and between the collimator and charge conversion subsystem (pressure region P2). First, UV and X-rays from several sources (including short wavelength UV backscattered from the interstellar helium and X-rays from astrophysical sources) can directly photo-ionise neutral gas. Second, these 
photons (including longer wavelength UV such as hydrogen Lyman- $\alpha$ ) can produce photoelectrons at the field shaping grids at the entrance to IBEX-Lo and the charge conversion foil on IBEX-Hi, and these photo-electrons can ionise residual gas atoms and molecules as they are accelerated across region $\mathrm{P} 2$ and through region $\mathrm{P} 1$. In pressure region $\mathrm{P} 2$, these ions are created in an electric field that accelerates them towards the conversion subsystem. A fraction of these ions can enter the charge conversion subsystem within the energy passband of the ESA subsystem and can therefore reach the detector subsystem. In pressure region P1, the ions are created nearly at rest at the potential of the collimator $(+\mathrm{HV})$. A fraction of these ions have a thermal velocity toward the collimator exit, and a larger fraction are accelerated out of the collimator exit by the fringe fields at the exit of the collimator's hexagonal channels. Therefore, these ions can escape into region P2. They are then accelerated by $+\mathrm{HV}$ and strike the conversion surface (IBEX-Lo) or the conversion foil (IBEX-Hi). These ions are composed almost entirely of water molecules. Although the molecules strike the conversion surface (IBEX-Lo) or transit the conversion foil (IBEX-Hi) at an energy greater than the highest energy passband of the sensors, the molecule is dissociated during charge conversion, and the molecular energy is partitioned to the atomic constituents based on the atomic mass. Thus, the energy of $\mathrm{H}$ after charge conversion is $1 / 18$ of the collimator voltage $+\mathrm{HV}$; if this voltage is $10 \mathrm{kV}$, then $\mathrm{H}$ will have an energy at the charge conversion surface or foil of $550 \mathrm{eV}$, which lies directly in the energy range of both sensors. Thus, such ions represent a significant background, and every effort has been made to minimize their generation and transmission further into the sensor. These suppression efforts will be discussed in detail in the following sections.

\section{External Backgrounds}

External background sources include all sources that result in an ion at the exit of the conversion subsystem that could masquerade as a signal ENA from the termination shock region. For IBEX-Lo a set of conversion surfaces where impacting atoms or ions are converted to negative ions upon reflection is used as conversion subsystem (Fuselier et al. 2009, this issue). IBEX-Hi uses a set of ultra-thin carbon foils, the charge conversion foils, through which atoms or ions pass, then exit positively ionised (Funsten et al. 2009, this issue).

\subsection{Ultraviolet Photon Background}

The Sun and stars are sources of UV photons, to which the detectors used in the IBEX sensors are sensitive. The onset of photo emission occurs at photon energies corresponding to the work function of the material and impurities at the material surface, but should be approximately 4 to $7 \mathrm{eV}$. For reference, the detection subsystems of both sensors utilise electron emission from carbon foils as an initial step to register passage of an ENA, with the electrons subsequently being registered by a microchannel plate (MCP) or CEM detector in the case of IBEX-Lo or IBEX-Hi, respectively. The work function of graphite is approximately $4.6 \mathrm{eV}$, which corresponds to an UV wavelength of $\lambda \approx 270 \mathrm{~nm}$ and defines the limit of sensitivity. Toward shorter wavelengths, the solar UV is a continuum spectrum with rapidly declining photon fluxes. At wavelengths below about $150 \mathrm{~nm}$ the solar spectrum is mostly a line spectrum, with large temporal fluctuations (Fröhlich and Lean 2004). Hydrogen Lyman- $\alpha(121.59 \mathrm{~nm}$, corresponding to approximately $10.2 \mathrm{eV})$ is a prominent UV line and can be detected directly by typical MCP or CEM detectors used in IBEX-Lo and IBEX$\mathrm{Hi}$, respectively, thus forward scattering of these photons to the detector subsystem must be efficiently suppressed. 
The interaction of molecular water with UV light results in molecular ions, fragment ions and neutrals (Huebner et al. 1992). The ionisation potential for the formation of $\mathrm{H}_{2} \mathrm{O}^{+}$ is about $12.62 \mathrm{eV}$, which corresponds to $\lambda=98.27 \mathrm{~nm}$ (Tonkyn et al. 1991; Merkt et al. 1998), and the thresholds for ion fragments resulting from the interaction of $\mathrm{H}_{2} \mathrm{O}$ molecules with UV photons are between 18.1 and $18.7 \mathrm{eV}$ (Huebner et al. 1992).

For a solar spectrum, the photo-ionisation rate of $\mathrm{H}_{2} \mathrm{O}^{+}$at $1 \mathrm{AU}$ is in the range (3.31$8.28) \cdot 10^{-7} \mathrm{~s}^{-1}$ for quiet and active Sun, respectively (Huebner et al. 1992). Using this solar photo-ionisation rate for a pressure of $10^{-8}$ mbar (assumed to be mostly $\mathrm{H}_{2} \mathrm{O}$ ) in the pressure region $\mathrm{P} 2$ results in about 60,000 ions $\mathrm{s}^{-1}$ being produced in region $\mathrm{P} 2$ if the sensor would be pointed directly at the Sun. Since IBEX is not viewing the Sun, this number is prorated to the expected UV fluxes of the sky, which results in the formation of about 0.1 ions/s, which are accelerated up to $10 \mathrm{kV}$ into the sensor onto the conversion surface. This is an overestimation, because the interstellar UV spectrum is composed mostly of the Lyman- $\alpha$ and $\mathrm{He} \mathrm{I}$ lines. In the following we will calculate the ionisation rates for the $\mathrm{He} I$ line for the background evaluation.

Fortunately, the H Lyman- $\alpha$ line is not energetic enough to ionise most of the atoms and molecules in a typical residual gas (i.e., in the collimator system at the sensor entrance), thus it does not create a direct ion background. However, there are ion background sources arising from the Lyman- $\alpha$, which will be discussed below. Another prominent UV line is the $\mathrm{He}$ I line at $\lambda=58.433 \mathrm{~nm}$ (corresponding to an energy of $21.22 \mathrm{eV}$ ), which is energetic enough to ionise most of the atomic and molecular species that might be present in the residual gas. Thus, He I photon fluxes have to be examined in more detail.

A coincident event measured in the detector subsystem requires two detections within a prescribed time window. Because photons scattered into the detector subsystem have sufficient energy to generate only one photo-electron, a coincident event in the detector subsystem requires the random occurrence of multiple photons generating photo-electrons that are detected by multiple detectors within the time window. This detection is called accidental coincidence, because the photons are not related to each other. A recent example of such an accidental coincidence background by UV photons has been discussed by Galli et al. (2006) in detail. In addition to the ion background from photo-ionisation, which may create an ion that is detected directly, there is photon stimulated desorption (PSD) in which UV photons will release atoms, molecules, and ions from exposed surfaces, causing a small particle background. This release of neutral particles adds to the local residual gas, which can be photo-ionised as a second step. Ions generated by PSD may be detected directly. Photon stimulated desorption is highly effective for releasing adsorbed water layers on surfaces for UV wavelengths $\leq 250 \mathrm{~nm}$ and is used in vacuum technology to clean high vacuum systems.

Resonant scattering of solar photons on interstellar hydrogen and helium causes the entire sky to be the source of Lyman- $\alpha$ and He I photons. Also, the geocorona is a source of these UV photons with about $1 \%$ of the solar flux, which has to be considered when assessing the UV background. Therefore, efficient UV suppression was needed for IBEX, although the IBEX sensors are never pointed toward the Sun.

The Lyman- $\alpha$ background from solar photons scattered at the interstellar hydrogen in interplanetary space is in the range from 400 to $900 R\left(1 R=1 / 4 \pi \cdot 10^{6}\right.$ photons $\mathrm{cm}^{-2} \mathrm{sr}^{-1} \mathrm{~s}^{-1}$, Baker and Romick 1976), depending on solar activity and look direction (Quémerais et al. 2006). The brightness of UV bright stars, which are clustered around the galactic plane, reaches up to $3000 R$. Figure 3 shows a map of the UV sky in galactic coordinates, recorded with the SWAN instrument on SOHO (Bertaux et al. 1995). Since UV bright stars are localised sources they can be identified in the recorded ENA maps and eliminated if necessary. 


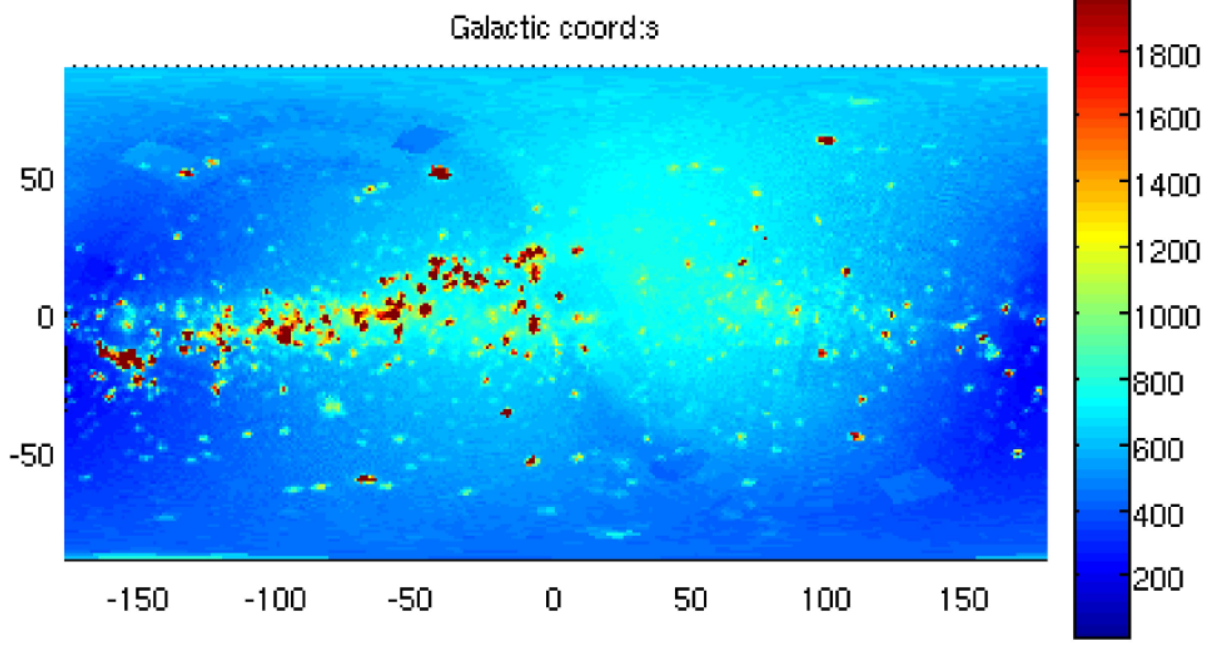

Fig. 3 SOHO/SWAN FUV fluxes (from 115 to $180 \mathrm{~nm}$, including Lyman- $\alpha$ ) for the full sky in galactic longitude and latitude (Bertaux et al. 1995). The bright regions ( $\geq 2 \mathrm{kR}$ ) near zero degrees galactic latitude are UV stars in the galactic plane in the direction of the galactic centre

The flux of $\mathrm{He} \mathrm{I}(\lambda=58.433 \mathrm{~nm})$ at $1 \mathrm{AU}$ is known from observations of interstellar He. According to Vallerga et al. (2004) the flux at 1 AU exactly downwind along the He focusing cone is about $\Phi_{\mathrm{HeI}}=7 R$ during solar minimum. Outside the focussing cone the typical He I flux is about $\Phi_{\mathrm{HeI}}=2 R$. In the following we will use $\Phi_{\mathrm{HeI}}=10 R$ as maximum illumination to obtain an upper limit. Moreover, IBEX viewing will at best be perpendicular to the focussing cone at $1 \mathrm{AU}$.

The rate of ions produced via photo-ionisation by the He I line in region $\mathrm{P} 2$ as shown in Fig. 2 is given by

$$
Q_{\mathrm{HeI}, 2}=A \cdot \Delta l \cdot \frac{p_{2}}{k_{B} T} \cdot \Phi_{\mathrm{Hel}} \cdot \Delta \Omega \cdot T_{\mathrm{col}} \cdot \sigma_{\mathrm{HeI}}
$$

where $A$ is the aperture area, $\Delta l$ is the effective length over which ions are generated with the correct energy for the energy passband of the actual ESA step, $p_{2}$ is the residual gas pressure in region P2 (see Fig. 2), $k_{B}$ is the Boltzmann constant, and $T$ is the gas temperature, i.e., the sensor temperature during laboratory testing. For the aperture area we take a value of $A=150 \mathrm{~cm}^{2}$; the actual aperture areas of IBEX-Lo and IBEX-Hi are a little bit different, which is not important for the calculations performed here. $\Phi_{\mathrm{HeI}}$ is the ionising He I photon flux, $\Delta \Omega$ is the FOV from the region $\mathrm{P} 2$ to the outside $\left(7^{\circ} \times 7^{\circ}\right.$, which gives $\Delta \Omega=0.0149 \mathrm{sr}), T_{\text {col }}$ is the collimator transmission $\left(T_{\text {col }}=0.69\right)$, and $\sigma_{\mathrm{HeI}}$ is the photoionisation cross section. For water the ionisation cross section is $2 \cdot 10^{-17} \mathrm{~cm}^{2}$ at $58.4 \mathrm{~nm}$ (Berkowitz 2002). Using a pressure of $p_{2}=10^{-8} \mathrm{mbar}$ in the pressure region $\mathrm{P} 2$ (a conservative estimate for the flight situation) and a He I photon flux of $\Phi_{\mathrm{HeI}}=10 R$ (again a conservatively large flux), the background rate $Q_{\text {HeI,2 }}$ was evaluated. Table 1 shows these results for all energy steps of IBEX-Lo and IBEX-Hi. These background fluxes are $\mathrm{H}_{2} \mathrm{O}^{+}$ ions at the front of the conversion surface (IBEX-Lo) or the conversion foil (IBEX-Hi), which eventually masquerade as ENA signals. Fortunately, the estimated fluxes are significantly lower than the requirement and therefore they are of no concern. 
Table 1 Calculated background rate for each of the IBEX energy channels: $Q_{\mathrm{HeI}, 1}$ and $Q_{\mathrm{HeI}, 2}$ for He I photo-ionisation in regions $\mathrm{P} 1$ and $\mathrm{P} 2$, respectively (rates for a pressure of $10^{-8}$ mbar at P1 and P2); $Q_{\mathrm{Ly} \alpha, 1}$ and $Q_{\mathrm{Ly} \alpha, 2}$ for electron impact ionisation induced by photo-electrons from Lyman- $\alpha$ light in regions $\mathrm{P} 1$ and $\mathrm{P} 2$, respectively (rates for a pressure of $10^{-8}$ mbar at $\mathrm{P} 1$ and $\mathrm{P} 2$ )

\begin{tabular}{|c|c|c|c|c|c|c|c|}
\hline Sensor & $\begin{array}{l}\text { Energy } \\
\text { channel }\end{array}$ & $\begin{array}{l}\text { Centre } \\
\text { energy }[\mathrm{keV}]\end{array}$ & $\Delta l[\mathrm{~cm}]$ & $\begin{array}{l}Q_{\mathrm{HeI}, 2} \\
\mathrm{H}_{2} \mathrm{O}^{+} \\
\text {rate }[\mathrm{cnt} / \mathrm{s}]\end{array}$ & $\begin{array}{l}Q_{\mathrm{HeI}, 1} \\
\mathrm{H}_{2} \mathrm{O}^{+} \\
\text {rate [cts/s] }\end{array}$ & $\begin{array}{l}Q_{\mathrm{Ly} \alpha, 2} \\
\mathrm{H}_{2} \mathrm{O}^{+} \\
\text {rate [cts/s] }\end{array}$ & $\begin{array}{l}Q_{\mathrm{Ly} \alpha, 1} \\
\mathrm{H}_{2} \mathrm{O}^{+} \\
\text {rate [cts/s] }\end{array}$ \\
\hline IBEX-Lo & 1 & 0.015 & 0.001 & $8.3 \cdot 10^{-6}$ & - & $3.2 \cdot 10^{-6}$ & - \\
\hline IBEX-Lo & 2 & 0.029 & 0.003 & $1.7 \cdot 10^{-5}$ & - & $6.4 \cdot 10^{-6}$ & - \\
\hline IBEX-Lo & 3 & 0.056 & 0.005 & $3.2 \cdot 10^{-5}$ & - & $1.2 \cdot 10^{-5}$ & - \\
\hline IBEX-Lo & 4 & 0.107 & 0.010 & $6.3 \cdot 10^{-5}$ & - & $2.4 \cdot 10^{-5}$ & - \\
\hline IBEX-Lo & 5 & 0.208 & 0.019 & $1.2 \cdot 10^{-4}$ & - & $4.5 \cdot 10^{-5}$ & - \\
\hline IBEX-Lo & 6 & 0.403 & 0.036 & $2.4 \cdot 10^{-4}$ & $6.5 \cdot 10^{-3}$ & $9.0 \cdot 10^{-5}$ & $4.9 \cdot 10^{-3}$ \\
\hline IBEX-Lo & 7 & 0.781 & 0.070 & $4.7 \cdot 10^{-4}$ & - & $1.7 \cdot 10^{-4}$ & - \\
\hline IBEX-Lo & 8 & 1.515 & 0.136 & $9.0 \cdot 10^{-4}$ & - & $3.3 \cdot 10^{-4}$ & - \\
\hline IBEX-Hi & 1 & 0.425 & 0.038 & $2.8 \cdot 10^{-4}$ & $1.3 \cdot 10^{-2}$ & $9.4 \cdot 10^{-5}$ & $2.4 \cdot 10^{-3}$ \\
\hline IBEX-Hi & 2 & 0.815 & 0.073 & $4.8 \cdot 10^{-4}$ & - & $1.8 \cdot 10^{-4}$ & - \\
\hline IBEX-Hi & 3 & 1.375 & 0.124 & $8.3 \cdot 10^{-4}$ & - & $3.0 \cdot 10^{-4}$ & - \\
\hline IBEX-Hi & 4 & 2.250 & 0.203 & $1.4 \cdot 10^{-3}$ & - & $4.9 \cdot 10^{-4}$ & - \\
\hline IBEX-Hi & 5 & 3.075 & 0.277 & $1.8 \cdot 10^{-3}$ & - & $6.8 \cdot 10^{-4}$ & - \\
\hline IBEX-Hi & 6 & 4.950 & 0.446 & $2.9 \cdot 10^{-3}$ & - & $1.1 \cdot 10^{-3}$ & - \\
\hline
\end{tabular}

The effective length $\Delta l$ is computed from the length of the electric field region, the total voltage across the gap, and the energy pass band of the analyser as

$$
\Delta l=h \frac{E}{+\mathrm{HV}} \cdot\left(\frac{\Delta E}{E}\right)_{\mathrm{ESA}}
$$

where $h$ is the minimum gap between $+\mathrm{HV}$ and ground $(h=10.0 \mathrm{~mm}),+\mathrm{HV}$ is the collimator bias $(+\mathrm{HV}=10.0 \mathrm{kV}$, nominally), $E$ is the centre energy of the energy passband of interest, and $\Delta E / E$ is the energy resolution of the ESA (for IBEX-Lo $\Delta E / E \approx 0.8$, for IBEX-Hi $\Delta E / E \approx 0.45-0.65$ depending on energy). $\Delta l$ linearly scales with the centre energy of the ESA passband and reaches its maximum at the highest energy ( $E=4.9 \mathrm{keV}$ for IBEX-Hi, energy step 6). For this energy $\Delta l=0.45 \mathrm{~cm}$. For comparison, $\Delta l=0.14 \mathrm{~cm}$ for the highest energy step of IBEX-Lo ( $E=1.5 \mathrm{keV}$, energy step 8). Table 1 also shows the effective length $\Delta l$ for all energy steps of IBEX-Lo and IBEX-Hi. The background fluxes $Q_{\text {SW,E }}$ are $\mathrm{H}_{2} \mathrm{O}^{+}$at the front of the conversion surface (IBEX-Lo) or the conversion foil (IBEX-Hi) for each energy step of IBEX-Lo and IBEX-Hi.

Photo-electrons can be emitted in region $\mathrm{P} 2$ by photon impact on exposed surfaces of the charge conversion subsystem (see Fig. 2). These photo-electrons are then accelerated back towards the collimator that lies at $+10 \mathrm{kV}$, and in their transit, they can ionise the neutrals in the P2 region. Of particular concern in IBEX-Hi is the production of photo-electrons off the large area consisting of conversion foils and foil frames, and their acceleration back into the $\mathrm{P} 2$ region. For this reason, a high transmission Ni grid is located between the conversion foils and the collimator and is biased to $-300 \mathrm{~V}$ to suppress photo-electrons back to the foils and foil frames. This grid reduces the flux of ions generated by photo-electrons from 
the foils and foil frames by a factor of approximately 20 . There is no similar issue for IBEXLo because there are only high transmission grids between the region P2 and the conversion surface (electrons produced on the conversion surface are suppressed by the magnets in that region).

As a result of these preventative measures, the only photo-electrons that can ionise ions in region P2 are generated at the grids between the collimator and charge conversion subsystem. Since these grids are designed to have high transmission $\left(T_{\text {grid }}>95 \%\right)$, the amount of surface area available for photo-electron production is minimised, although they span the same area as the aperture. We can calculate the rate of ions produced by photo-electron impact using

$$
Q_{L y \alpha, 2}=A \cdot \Delta l \cdot \frac{p_{2}}{k_{B} T} \cdot \Phi_{\mathrm{ele}} \cdot \Delta \Omega \cdot T_{\mathrm{col}} \cdot \sigma_{2} \cdot\left(1-T_{\text {grid }}\right)
$$

where $\Phi_{\text {ele }}$ is the ionising flux of photo-electrons off the high transmission grids behind the collimator (see Fuselier et al. 2009, this issue for details of the sensor design), $T_{\text {grid }}$ is the grid transmission, and $\sigma_{e}$ is the electron ionisation cross section $\left(\sigma_{e}=1.25 \cdot 10^{-16} \mathrm{~cm}^{2}\right.$, the average between $100 \mathrm{eV}$ and $10 \mathrm{keV}$ electrons), and the other symbols as before. For the IBEX observations we only need to consider the Lyman- $\alpha$ flux of the night sky, for which we take $\Phi_{L y \alpha}=1200 R$ (as a conservative value). For the photo-electron flux we get $\Phi_{\text {ele }}=\Phi_{L y \alpha} \cdot \varepsilon$, with $\varepsilon$ the efficiency of photo-electron emission. Using $\varepsilon=0.01$ (Krolikowski and Spicer 1970 ), we get $\Phi_{\text {ele }}=1.9 \cdot 10^{6}$ electrons $/\left(\mathrm{cm}^{2} \mathrm{~s} \mathrm{sr}\right)$. Again, these background fluxes are much lower than the requirement and Table 1 shows the calculated results for this background.

As with region $\mathrm{P} 2$, there is photo-ionisation in region P1 within the collimator (see Fig. 2), which can be expressed as

$$
Q_{\mathrm{HeI}, 1}=A \cdot \Delta l_{P 1} \cdot \frac{p_{1}}{k_{B} T} \cdot \Phi_{\mathrm{HeI}} \cdot \Delta \Omega \cdot T_{\mathrm{col}} \cdot \sigma_{\mathrm{HeI}} \cdot 2 P_{\mathrm{Hit}}
$$

where $\Delta l_{P 1}$ is the size of region $\mathrm{P} 1\left(\Delta l_{P 1}=1 \mathrm{~cm}\right), p_{1}$ is the residual gas pressure in region $\mathrm{P} 1, \Phi_{\mathrm{HeI}}$ is the ionising flux of He I photons, and the other symbols as before. The factor of 2 in (4) accounts for the 2 hydrogen atoms in a water molecule, which have an equal probability of exiting the conversion foil or leaving the conversion surface with a positive or negative charge, respectively. $P_{\mathrm{Hit}}$ is the probability that the ion created in region P1 will hit the conversion surface (IBEX-Lo: $P_{\mathrm{Hit}}=0.5$ ) or the conversion foil (IBEX-Hi: $P_{\mathrm{Hit}}=1.0$ ).

Using $p_{1}=10^{-8}$ mbar pressure in the pressure region $\mathrm{P} 1$ and a He I photon flux of $\Phi_{\mathrm{HeI}}=10 R$, the background rate $Q_{\mathrm{HeI}, 1}$ was evaluated. Ions created in region P1 may drift further into the sensor and are accelerated by the collimator potential to $10 \mathrm{keV}$, nominally. Water ions impinging on the conversion surface or at the conversion foil at $10 \mathrm{keV}$ energy will disintegrate into its atomic constituents. The created atomic constituents, $\mathrm{H}$ and $\mathrm{O}$, will leave the surface with an energy proportional to their mass (and a small energy loss). Thus, only hydrogen with about $550 \mathrm{eV}$ will be inside the energy range of IBEX-Lo and IBEX-Hi. Table 1 shows these two results for the pertaining energy steps of IBEX-Lo and IBEX-Hi. The expected background is smaller than the value in the requirement. Given that we used a rather high $\mathrm{He}$ I flux for our estimates, the closeness of this background to the requirement is not a concern.

As with region P2, there is ionisation by photo-electrons in region P1 (see Fig. 2), $Q_{L y \alpha, 1}$, for which (3) also applies with $\Delta l=\Delta l_{P 1}$. Again, Table 1 shows the calculated results for this background, which is found to be significantly below the requirement and thus of no concern. 

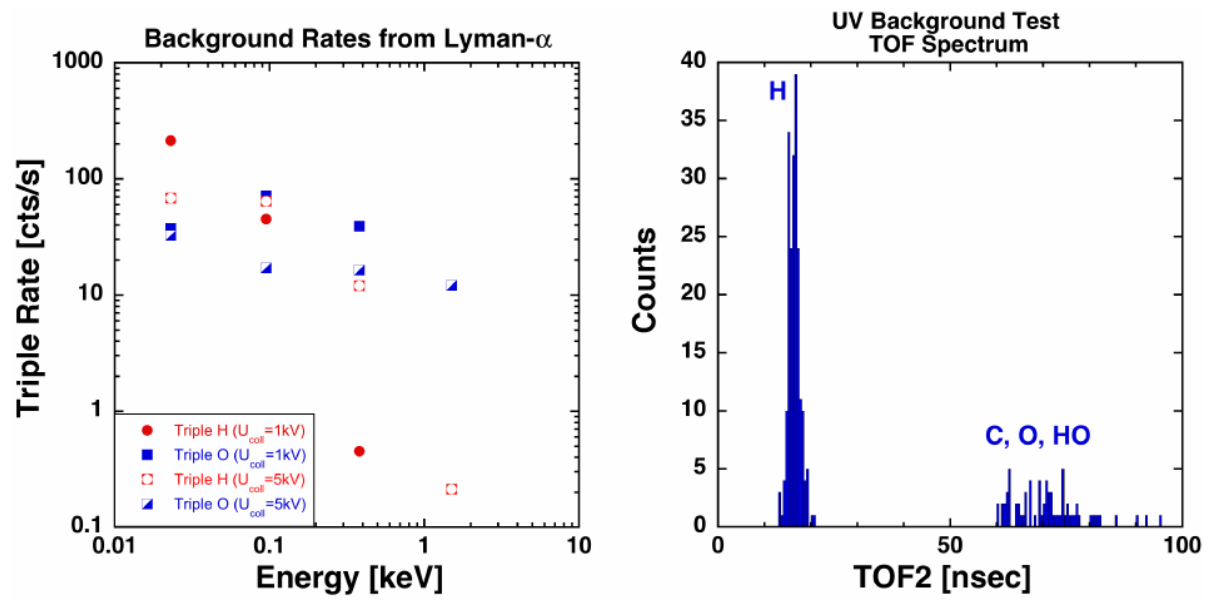

Fig. 4 Results of the UV illumination of IBEX-Lo. Left: Triple rates observed for H, C, and O for two collimator voltages. Right: Typical mass spectrum recorded during UV tests. Further details are given in the text

\subsubsection{The IBEX-Lo UV Background}

During the IBEX-Lo calibration campaign a UV background test was performed. For that test an excimer VUV lamp (Ushio, Japan) operating at $126 \mathrm{~nm}$ was used. A section of the IBEX-Lo entrance was illuminated with $7.2 \cdot 10^{13}$ photons/s, which corresponds to $1.2 \cdot 10^{6}$ times the Lyman- $\alpha$ background rate expected in flight $\left(5.8 \cdot 10^{7}\right.$ photons/s) entering IBEXLo. The wavelength of $126 \mathrm{~nm}$ (close to Lyman- $\alpha$ ) used during the UV background test is too long to cause direct ionisation of residual gas molecules. As discussed above, released photo-electrons will cause ionisation resulting in an ion flux of $Q_{L y \alpha, 2}$ from region P2, and photon stimulated desorption of neutrals and ions will take place as well. Figure 4, left panel, shows the triple TOF rates of the detected species as a function of particle energy and collimator voltage recorded during the UV background test. We find that the UV induced background rates depend on the ESA step and the collimator potential, as expected for the $Q_{L y \alpha, 2}$ background ion flux. The mass spectrum given in Fig. 4, right panel, shows a prominent hydrogen peak indicative of water ions, and a broader peak, which is a mixture of $\mathrm{C}$, $\mathrm{O}$, and HO. The ions (mostly water) produced by ionisation through photo-electrons are accelerated toward the charge conversion system to substantial energies and hit the conversion surface where they break up and also may cause sputtering of surface adsorbates. This fully explains the observed mass spectrum shown in Fig. 4.

However, the observed dependence on energy and collimator voltage does not agree with the prediction, since (3) shows an increase in this background signal (see also Table 1) and we observe a decrease with energy. The measured energy dependence of the background fluxes suggests sputtering from the exit structures of the collimator as the main source rather than electron impact ionisation. In addition, a test was run during which the residual gas pressure in the test chamber was deliberately increased by a factor of 2 to see whether most of the UV generated positive ions come from the residual gas in the pressure region $\mathrm{P} 2$ or from the exit structures of the collimator. No significant change of the background flux with the ambient gas pressure was seen. This is consistent with the collimator exit structure as the main source, i.e., from electron- and photon-induced desorption of positive ions from 
the last collimator grids. Therefore, the observed particle rate can be taken as a measure of the expected background arising from the Lyman- $\alpha$.

Taking the measured UV background rates (Fig. 4, left panel) prorated to the situation during the measurements in space we can calculate backwards to derive the "equivalent" neutral particle fluxes on the conversion surface. Using a TOF efficiency of 0.2 (for triples), an ESA transmission of 0.5, an ionisation efficiency of 0.04 for hydrogen (Wurz et al. 2006) and a reflection probability of 0.06 , we get a total efficiency of $2.7 \cdot 10^{-4}$ at $400 \mathrm{eV}$ to convert the triple rates to a flux on the conversion surface. Thus, the measured triple rate of $\mathrm{H}$ for energy bin \#6 (about $400 \mathrm{eV}$, see Fig. 4) converts to an equivalent flux of $0.030 \mathrm{~s}^{-1}$, taking into account the ratio of UV fluxes. A comparison of the expected signal with the measured background rates is given in Fig. 5. For energy bin \#4 (about $100 \mathrm{eV}$ ) we get $0.24 \mathrm{~s}^{-1}$ hydrogen ENAs background flux, compared to 10 to 100 hydrogen ENAs per second of the ENA signal from the termination shock region. For most of the energy range the expected background resulting from Lyman- $\alpha$ illumination is significantly below the expected ENA signal, and the signal-to-noise ratio is comfortably large (see Fig. 5). Depending on the actual shape of the energy spectrum of the observed ENAs the signal-to-noise ratio becomes very small only at the lowest two energy bins.

The background due to Lyman- $\alpha$ photons attributed to photo-electron release and subsequent electron impact ionisation of molecules in the pressure regions P1 and P2 has been calculated above and is given in Table 1 . These values are much lower than the measured ones, even when considering the higher pressure of $10^{-7}$ mbar during the measurements. Thus, the background attributed to photo-electron release and subsequent electron impact ionisation can be neglected in further analysis, and only the measured rates given in Fig. 5 need to be considered.

\subsubsection{The IBEX-Hi Ultraviolet Background}

IBEX-Hi was also tested for response to UV light, particularly to quantify the background created by Lyman- $\alpha$ light. During the calibration campaign, an Ar-purged deuterium lamp, followed by two notch filters used to maximize the fraction of Hydrogen Lyman- $\alpha$ $(121.6 \mathrm{~nm})$, and a $\mathrm{MgF}_{2}$ window directly illuminated the conversion foils. The photon rate at the foils was $\sim 4 \cdot 10^{10} \mathrm{~s}^{-1}$ as measured using a calibrated UV photodiode (Korde et al. 2003). The photon-induced ion production was observed when the collimator was biased to $+10 \mathrm{kV}$, yielding the following information: individual count rates in CEMs A, B, and $\mathrm{C}$ of 17,12 , and $1.6 \mathrm{~s}^{-1}$ respectively; a total double coincidence rate of about $0.1 \mathrm{~s}^{-1}$; and a total triple coincidence rate below $0.05 \mathrm{~s}^{-1}$. During these tests no voltage was applied to the photo-electron suppression grid to maximise the effect. Unfortunately, experimental difficulties during the calibration campaign prevented us from measuring this background at increasing voltages on the suppression grid, therefore the present background numbers are an upper limit of what is expected in space. As expected, the double and triple coincident rates dropped to the internal background levels when the collimator voltage was switched from $+10 \mathrm{kV}$ to $0 \mathrm{~V}$. Adjusted to the interstellar Lyman- $\alpha$ photon flux, the total double coincidence rate becomes $1.5 \cdot 10^{-4} \mathrm{~s}^{-1}$ and the total triple coincidence rate becomes $7.5 \cdot 10^{-5}$ $\mathrm{s}^{-1}$, which are in reasonable agreement with the expected values (see Table 1).

We also tested the response of the IBaM to UV light using a UV krypton line lamp (Resonance Ltd., model KrLM-L, serial number R399) with 116.5 and $123.6 \mathrm{~nm}$ wavelengths and intensity close to 1 solar unit of H Lyman- $\alpha$ at the aperture. We varied the angle between the centre of the IBaM FOV and the direction to the lamp. The lamp then shines straight into the IBaM when this angle is equal to $0^{\circ}$. Figure 6 shows the count rate as a function of this 


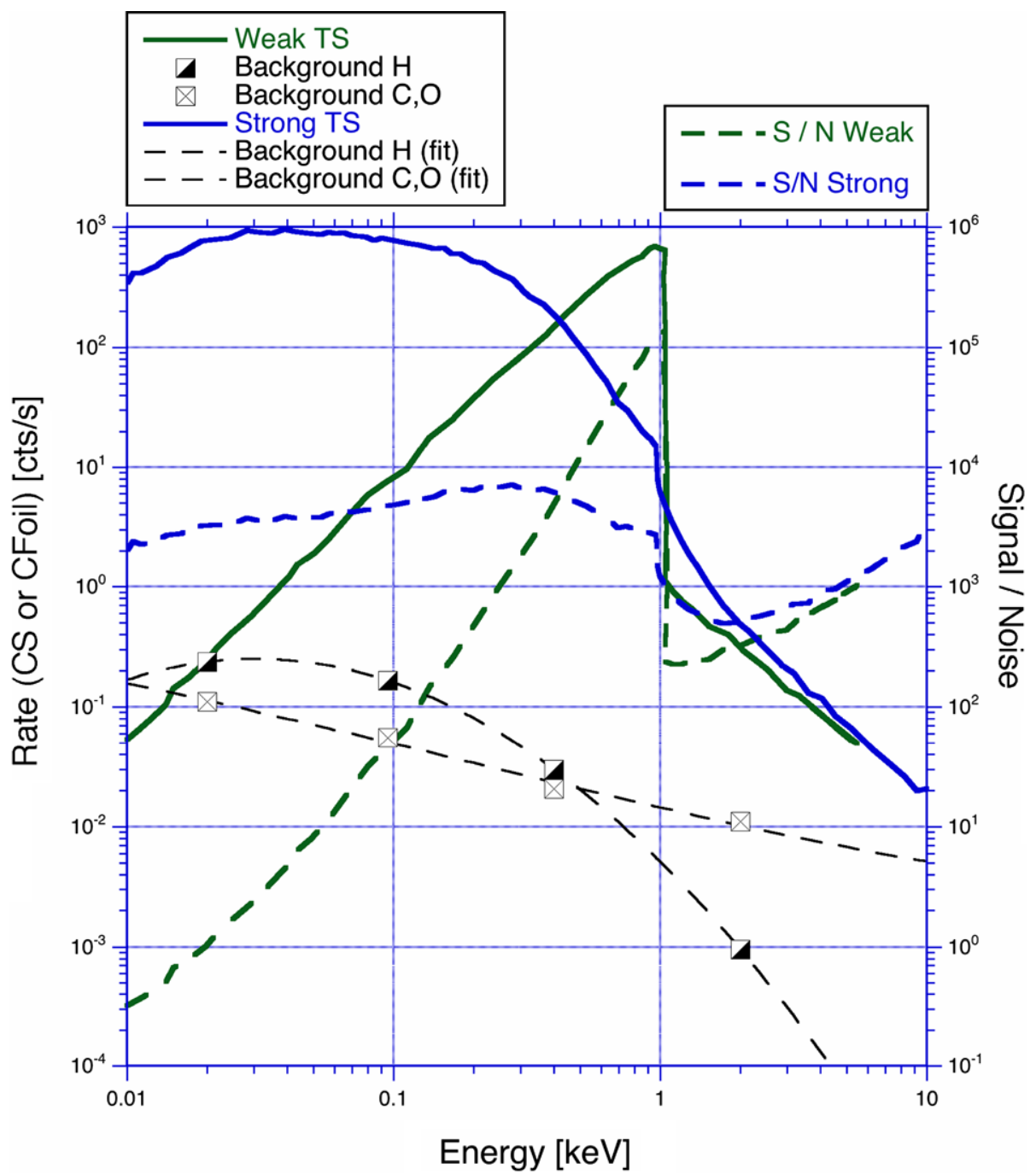

Fig. 5 Measured IBEX-Lo particle background (triples) on the conversion surface as a result of Lyman- $\alpha$ flux compared to the expected signal on the conversion foil or the conversion surface. The expected ENA signal is based on the model by Gruntman et al. (2000)

angle, revealing the expected peak at $0^{\circ}$ look direction with a count rate of about $600 \mathrm{~s}^{-1}$ when the UV radiation is shining straight into the IBaM's collimator aperture. For this test, the IBaM was placed in the engineering ground support equipment. An additional light leak test was performed during the IBEX cross calibration when the IBEX-Hi sensor was in its flight configuration. We did not find any light leaks for the beam monitor, which verified that the light tight design worked as expected.

Prorating the maximum background rate of about $600 \mathrm{~s}^{-1}$, when UV goes straight through the collimator, from the solar UV fluxes to the interstellar UV fluxes gives a background rate of $9 \cdot 10^{-4} \mathrm{~s}^{-1}$. Assuming that the UV intensity reflected by the Earth is roughly $1 / 100$ of the solar H Lyman- $\alpha$ intensity at $1 \mathrm{AU}\left(\sim 3 \cdot 10^{11}\right.$ photons $\left.\mathrm{cm}^{-2} \mathrm{~s}^{-1}\right)$, the max- 
Fig. 6 UV induced background in the IBEX Background Monitor as a function of the angle from the look direction, which is at $0^{\circ}$

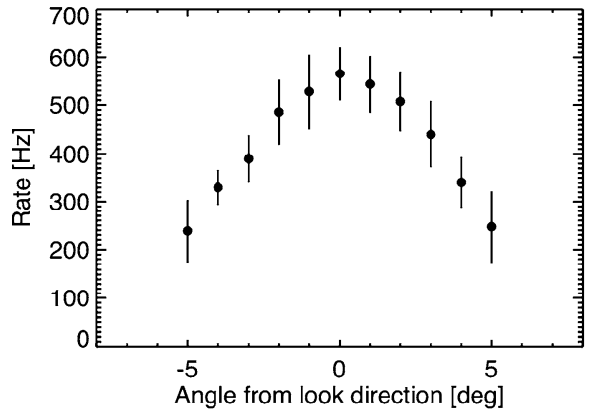

imum H Lyman- $\alpha$ intensity when the IBaM will look straight at the Earth will be about $3 \cdot 10^{9}$ photons $\mathrm{cm}^{-2} \mathrm{~s}^{-1}$. Thus, we can expect a background count rate of a maximum of approximately $5.5 \mathrm{~s}^{-1}$ from the geocorona.

\subsection{Local Ion Populations}

Background ENAs are generated from the charge exchange of ambient plasma ions with outgassed atoms and molecules from the spacecraft. Ambient ions, if not screened off by the collimator bias, could strike the collimator grids, charge exchange into neutrals, and then continue on to the conversion subsystem masquerading as ENAs. These background signals are indistinguishable from the signal. The fluxes of background ions depend on the location of the IBEX spacecraft. The fluxes are highest within the magnetosheath and foreshock regions with substantial omni-directional populations of energetic ions. Since both sensor FOVs are always pointed about $90^{\circ}$ away from the Sun, and thus from the bulk solar wind flow direction, the solar wind ion population does not play a role for this background, which will be shown below.

Aside from the generation of neutrals on the edges of the grids in the collimator, the production of neutrals locally near the spacecraft that masquerade as ENAs from the distant heliosphere depends on the outgassing pressure from the spacecraft and the ion flux. Outgassing products from the sensor typically consist of water and associated molecules. The conservative estimate of this background ENA differential flux, $j_{0}$, is

$$
j_{0}=\int_{r_{0}}^{\infty} \mathrm{d} r \cdot n(r) \cdot \sigma_{\mathrm{H}} \cdot J_{I}
$$

where $n$ is the number density of outgassing species (that decreases as $r^{-2}$ with distance $r$ from the spacecraft), $\sigma_{\mathrm{H}}$ is the charge exchange cross section of $\mathrm{H}^{+}$on water and water related species, and $j_{I}$ is the ambient ion differential flux. The integral is performed from the spacecraft surface, $r_{0}$, to infinity. The neutral density is expressed as $n(r)=n_{S / C}\left(r_{S / C} / r\right)^{2}$ where $r_{S / C}$ is the spacecraft radius and $n_{S / C}$ is the density at the spacecraft surface, so the integral equation yields

$$
j_{0}=n_{S / C} \cdot r_{S / C} \cdot \sigma_{\mathrm{H}} \cdot j_{I}=\frac{p_{S / C}}{k_{B} T} \cdot r_{S / C} \cdot \sigma_{\mathrm{H}} \cdot j_{I}
$$

where $p_{S / C}$ is the pressure at the spacecraft surface, $\sigma_{\mathrm{H}}$ is the energy dependent charge exchange cross section of $\mathrm{H}^{+}$on $\mathrm{H}_{2}, \mathrm{O}_{2}, \mathrm{H}_{2} \mathrm{O}, r_{S / C}$ is the radius of the spacecraft $\left(r_{S / C} \approx\right.$ $50 \mathrm{~cm}$ ), and $j_{I}$ is the ion flux directed into the collimator. This estimate assumes that the 
Table 2 Neutral fluxes on the conversion surface or conversion foil due to charge exchange with spacecraft outgassed neutral gas for the IBEX energy channels: $Q_{\mathrm{CX}}$ for magnetosheath ions (rates for a pressure of $10^{-11}$ mbar near the spacecraft), $Q_{\mathrm{SW}, \mathrm{I}}$ for solar wind ions (rates for a pressure of $10^{-11}$ mbar near the spacecraft), $Q_{\mathrm{SW}, \mathrm{E}}$ for solar wind electrons from regions $\mathrm{P} 2$ and P1 (rates for a pressure of $10^{-8} \mathrm{mbar}$ at P1 and P2)

\begin{tabular}{lllllll}
\hline Sensor & $\begin{array}{l}\text { Energy } \\
\text { channel }\end{array}$ & Centre energy [keV] & $\begin{array}{l}Q_{\mathrm{CX}} \\
{[\mathrm{cnt} / \mathrm{s}]}\end{array}$ & $\begin{array}{l}Q_{\mathrm{SW}, \mathrm{I}} \\
{[\mathrm{cnt} / \mathrm{s}]}\end{array}$ & $\begin{array}{l}\mathrm{P} 2: Q_{\mathrm{SW}, \mathrm{E}} \\
{[\mathrm{cnt} / \mathrm{s}]}\end{array}$ & $\begin{array}{l}\mathrm{P} 1: Q_{\mathrm{SW}, \mathrm{E}} \\
{[\mathrm{cnt} / \mathrm{s}]}\end{array}$ \\
\hline IBEX-Lo & 1 & 0.015 & $3.8 \cdot 10^{-6}$ & $9.0 \cdot 10^{-3}$ & $3.4 \cdot 10^{-6}$ & - \\
IBEX-Lo & 2 & 0.029 & $1.4 \cdot 10^{-5}$ & $1.8 \cdot 10^{-3}$ & $6.6 \cdot 10^{-6}$ & - \\
IBEX-Lo & 3 & 0.056 & $3.1 \cdot 10^{-5}$ & $1.0 \cdot 10^{-4}$ & $1.3 \cdot 10^{-5}$ & - \\
IBEX-Lo & 4 & 0.107 & $7.5 \cdot 10^{-5}$ & $8.2 \cdot 10^{-7}$ & $2.5 \cdot 10^{-5}$ & - \\
IBEX-Lo & 5 & 0.208 & $1.9 \cdot 10^{-4}$ & $1.2 \cdot 10^{-10}$ & $4.8 \cdot 10^{-5}$ & - \\
IBEX-Lo & 6 & 0.403 & $1.7 \cdot 10^{-4}$ & $6.4 \cdot 10^{-18}$ & $9.0 \cdot 10^{-5}$ & $5.1 \cdot 10^{-3}$ \\
IBEX-Lo & 7 & 0.781 & $1.1 \cdot 10^{-4}$ & 0 & $1.8 \cdot 10^{-4}$ & - \\
IBEX-Lo & 8 & 1.515 & $5.4 \cdot 10^{-5}$ & 0 & $3.5 \cdot 10^{-4}$ & - \\
& & & $1.7 \cdot 10^{-4}$ & 0 & $1.0 \cdot 10^{-4}$ & $2.6 \cdot 10^{-3}$ \\
IBEX-Hi & 1 & 0.425 & $1.1 \cdot 10^{-4}$ & 0 & $1.9 \cdot 10^{-4}$ & - \\
IBEX-Hi & 2 & 0.815 & $5.4 \cdot 10^{-5}$ & 0 & $3.2 \cdot 10^{-4}$ & - \\
IBEX-Hi & 3 & 1.375 & $4.7 \cdot 10^{-5}$ & 0 & $5.2 \cdot 10^{-4}$ & - \\
IBEX-Hi & 4 & 2.250 & $3.5 \cdot 10^{-5}$ & 0 & $7.1 \cdot 10^{-4}$ & - \\
IBEX-Hi & 5 & 3.075 & $6.7 \cdot 10^{-6}$ & 0 & $1.1 \cdot 10^{-3}$ & - \\
IBEX-Hi & 6 & 4.950 & & & & \\
\hline
\end{tabular}

charge exchange process does not change the flight direction significantly, and the neutralised ion continues to propagate in the initial direction. This assumption is valid for the ion energies of concern for this calculation (see Fig. 7), but at ion energies of about $10 \mathrm{eV}$ and below this assumption is increasingly violated (Hodges and Breig 1991). The neutral flux $j_{0}$ represents a background into the collimator. Thus, the neutral flux onto the conversion surface or conversion foil arising from charge exchange is expressed as

$$
Q_{\mathrm{CX}}=A \cdot \Delta \Omega \cdot T_{\mathrm{col}} \cdot J_{0}=A \cdot \Delta \Omega \cdot T_{\mathrm{col}} \cdot \frac{p_{S / C}}{k_{B} T} \cdot r_{S / C} \cdot \sigma_{\mathrm{H}} \cdot j_{I} .
$$

After several months in space, we can assume the pressure of the spacecraft surface is $p_{S / C}=10^{-11}$ mbar, based on Rosetta measurements (Graf et al. 2008) and because IBEX is a much smaller spacecraft that has undergone stringent cleanliness control. For the magnetosheath (the worst case) we used the measured ion fluxes from the CODIF instrument on the Cluster mission for our analysis (shown in Fig. 7), which are similar to magnetosheath measurements reported earlier (Williams et al. 1988; Paschalidis et al. 1994). Table 2 shows the background fluxes on the conversion surface or conversion foil for the IBEX energy steps under these assumptions and using the cross sections of $\mathrm{H}^{+}$on water (Greenwood et al. 2000). These background fluxes are lower than the requirements (see Sect. 1.2) and are therefore no concern for the mission.

The strongest background contribution would originate from ambient ions striking the edges of the front collimator grid. Assuming that the entire edge area of the 50- $\mu \mathrm{m}$-thick grid is the cross-section for interaction, the resulting neutral-atom fluxes, after charge exchange on the surface from the expected ambient fluxes, would have presented an overwhelming background. This assessment led to the conclusion that all ions up to $10 \mathrm{keV}$ had to be 
Fig. 7 Spectra of magnetosheath ions at three different locations in the magnetosphere. Data are from CODIF/Cluster

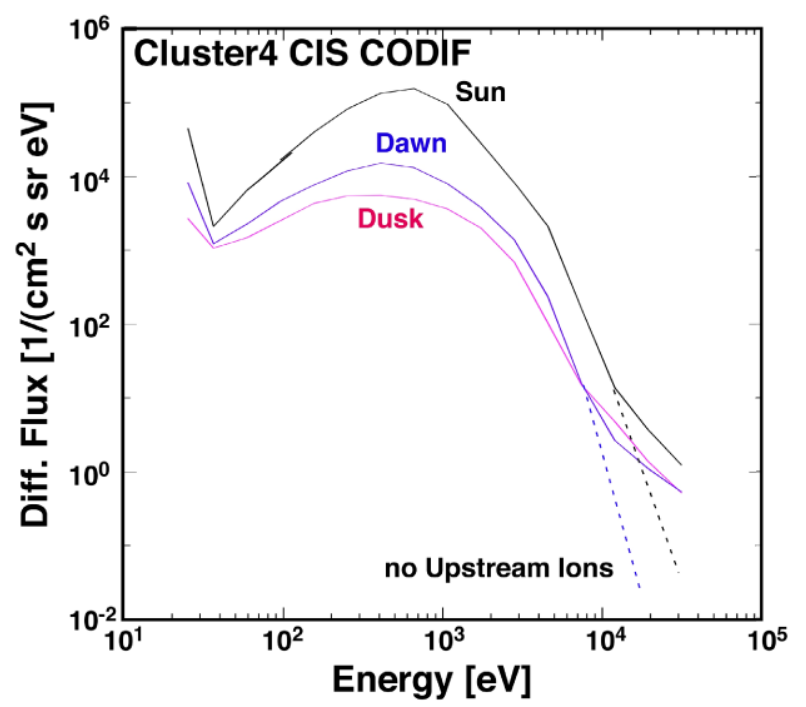

electrostatically rejected. The passage of ions between 10 and $16 \mathrm{keV}$ (the energy range of the sensors after decelerating these ions by the collimator potential) through the collimator had to be minimised. As a result background count rates could be reduced to well below the expected signal for most of the sensor energy range even in the magnetosheath.

Solar wind ions are a supersonic flow of mostly protons, about $4 \%$ alpha particles, and the total of heavier ions at the permil level. Solar wind velocities are in the range between 300 and $800 \mathrm{~km} / \mathrm{s}$ and Mach numbers range between 10 and 20. Since the respective FOVs of the IBEX sensors are pointed roughly perpendicular to the direction of the solar wind flow, there is very little ion flow toward the sensor apertures, therefore we expect only a small flux of neutralised solar wind background, $Q_{\mathrm{SW}, \mathrm{I}}$, directed toward the apertures of the IBEX sensors. We estimated this neutral background, $Q_{\mathrm{SW}, \mathrm{I}}$, using a Maxwellian-Boltzmann description of the solar wind velocity distribution in three dimensions. The evaluation of the distribution for the energy (i.e., velocity) intervals of the IBEX sensors gives the solar wind ion fluxes $j_{I}$ toward the entrance apertures, and applying (7) gives the neutral fluxes entering the IBEX sensors. We considered two cases: slow solar wind (with a solar wind speed of $v_{S W}=400 \mathrm{~km} / \mathrm{s}$, a proton density of $n_{S W}=8 \mathrm{~cm}^{-3}$, and a kinetic temperature of $v_{t h}=20 \mathrm{~km} / \mathrm{s}$, i.e., Mach number $\left.M=10\right)$ and fast solar wind $\left(v_{S W}=800 \mathrm{~km} / \mathrm{s}\right.$, a density of $n_{S W}=3 \mathrm{~cm}^{-3}$, and a kinetic temperature of $v_{t h}=40 \mathrm{~km} / \mathrm{s}$, i.e., $M=20$ ), but found that the results for the neutral background were very similar for these two cases, with the fast solar wind case resulting in a somewhat smaller neutral background. We found that only the lower energy channels of IBEX-Lo are affected by solar wind plasma and the background is considerably lower than the requirement. The estimates for this background for the slow solar wind case are given in Table 2.

\subsubsection{IBEX-Hi Background Monitor}

Ions with energies higher than the positive high voltage applied to the collimator, $+\mathrm{HV}$, have direct access to the ENA to ion conversion section. These ions will have the wrong energy for transmission through the ESA, but can cause release of particles by sputtering negative ions from the conversion surfaces or positive ions from the conversion foils. The other way 
for these ions to create a background signal (as discussed above) is if they scatter off the edge of a collimator grid, become neutral, and pass through the collimator to the ENA to ion conversion subsystem with energy less than their original energy, i.e., reduced by the collimator potential and by some energy loss from the scattering.

In the solar wind the proton flux above $10 \mathrm{keV}$ is very low and of no concern for the ENA measurements, as discussed above. However, if a spacecraft like IBEX is connected magnetically to the certain parts of the Earth's bow shock, creating a so-called upstream event, energetic particles from the bow shock can be observed far upstream in the solar wind (Sanderson et al. 1996). Figure 8 shows an example of an energy spectrum of an upstream event.

There are considerable proton fluxes above energies of $10 \mathrm{keV}$. Therefore, the IBaM was introduced to measure the ion fluxes above about $14 \mathrm{keV}$ to provide real-time information for this background source. The IBaM reports counts as a function of spin angle with the same resolution as IBEX-Hi and IBEX-Lo. This provides key information about the nature of the local ion environment (e.g., solar wind ions, magnetospheric ions, or ion fluxes through connection to the bow shock) and how to interpret this as a potential background in the ENA spectra and possibly cull the ENA data during these times.

\subsection{Local Electron Populations}

Unlike solar wind ions, solar wind electrons are subsonic, i.e., their mean thermal speed considerably exceeds the solar wind (ion) bulk speed. From observations it is known that the solar wind electron population is composed of three main components: a cold and almost isotropic collisional core; a hot, variably-skewed halo population, and, in fast solar wind, often a narrow strahl aligned with the magnetic field (Pilipp et al. 1987).

Electrons from the high-energy tail of the distribution of solar wind electrons penetrate through the collimator and enter region P2 (see Fig. 2). There, they will act as an additional ionisation source that creates ions in the $\mathrm{P} 2$ region. The rate of ions produced in this manner is

$$
Q_{S W, E}=A \cdot \Delta l \cdot \frac{p_{2}}{k_{B} T} \cdot \Phi_{S W, E} \cdot \Delta \Omega \cdot T_{\mathrm{col}} \cdot \sigma_{e}
$$

where $\Phi_{\mathrm{SW}, \mathrm{E}}$ is the high energy electron flux into the charge exchange region, and $\sigma_{e}$ is the electron ionisation cross section. The electron flux $\Phi_{\mathrm{SW}, \mathrm{E}}$ is region time dependent. As an estimate, we take an average flux from the solar wind as measured by the Ulysses solar wind electron spectrometer (McComas et al. 1992). The solar wind sensor measured up to $511 \mathrm{eV}$ and these electron fluxes were extrapolated to $600 \mathrm{eV}$. The differential energy fluxes represent the "average" flux observed over the mission. These fluxes were integrated to determine the electron number flux above $600 \mathrm{eV}$, which gives $1.5 \cdot 10^{4}$ electrons $/\left(\mathrm{cm}^{2} \mathrm{~s} \mathrm{sr}\right)$ above $700 \mathrm{eV}$. The electron impact ionisation cross section of water is $\sigma_{e}=2 \cdot 10^{-16} \mathrm{~cm}^{2}$ at $100 \mathrm{eV}$ and $\sigma_{e}=0.5 \cdot 10^{-16} \mathrm{~cm}^{2}$ at $10 \mathrm{keV}$. For the estimation of $Q_{\mathrm{SW}, \mathrm{E}}$ we used an average value of $\sigma_{e}=1.25 \cdot 10^{-16} \mathrm{~cm}^{2}$. The product $A \cdot \Delta l$ gives the volume in which the ions are created.

As with region P2, there is electron impact ionisation in region P1 (see Fig. 2), which can be expressed as

$$
Q_{S W, E}=A \cdot \Delta l_{P 1} \cdot \frac{p_{1}}{k_{B} T} \cdot \Phi_{S W, E} \cdot \Delta \Omega \cdot T_{\mathrm{col}} \cdot \sigma_{e} \cdot P_{\mathrm{Hit}} \cdot
$$

Again, the factor of 2 in (9) accounts for the 2 hydrogen atoms in a water molecule, which exhibit equal probability to leave the conversion foil with a positive or the conversion surface 


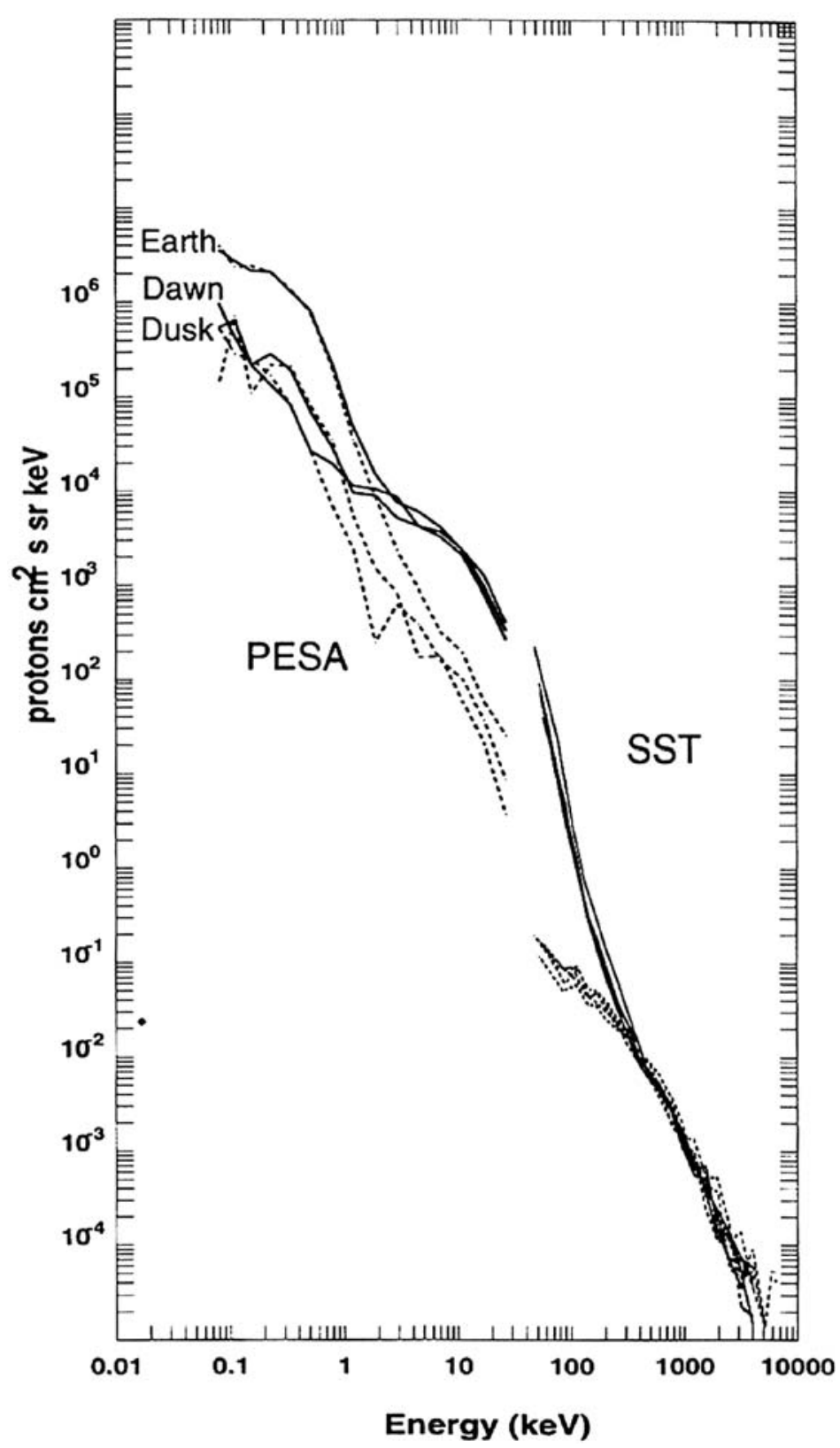

Fig. 8 Composite proton spectrum of upstream event measured by PESA and SST on WIND on 13 August 1995 (Sanderson et al. 1996)

with a negative charge. $P_{\mathrm{Hit}}$ is the probability that the ion created in region P1 will hit the conversion surface (IBEX-Lo: $P_{\mathrm{Hit}}=0.5$ ) or the conversion foil (IBEX-Hi: $P_{\mathrm{Hit}}=1.0$ ). Again, Table 2 shows the results for this background, which are found to be considerably below the requirement. 


\subsection{Penetrating Radiation}

Particles with very high energy (exceeding $1 \mathrm{MeV} /$ nucleon) can penetrate the thin walls of space instrumentation and cause background signals when hitting a detector. The terrestrial radiation belts are a source of such particles. However, the IBEX orbit is designed to have a low radiation dose from radiation belt particles (McComas et al. 2009, this issue), with the IBEX orbit being far from the near-Earth radiation environment during data acquisition. Data acquired while the IBEX spacecraft is inside the magnetosphere are not analysed.

Another source of highly energetic particles is cosmic rays, which consist of approximately $83 \%$ protons, $13 \%$ alpha particles, $1 \%$ heavier nuclei, and $3 \%$ electrons. The cosmic ray energy spectrum extends from a few $100 \mathrm{MeV}$ to energies in excess of $10^{20} \mathrm{eV}$ (McDonald and Ptuskin 2001). In the ecliptic plane the intensity of cosmic ray particles depends on the solar cycle, with the modulation being anti-correlated with solar activity. The isotropic flux of galactic cosmic rays at $1 \mathrm{AU}$ is 4 protons $/\left(\mathrm{cm}^{2} \mathrm{~s}\right)$ at sunspot minimum and 2 protons $/\left(\mathrm{cm}^{2} \mathrm{~s}\right)$ at sunspot maximum. The cosmic ray flux is measured by a variety of spacecraft, thus measurements are available if needed. In the energy range between $1 \mathrm{MeV} /$ nucl and $\leq 70 \mathrm{MeV} /$ nucleon there are, in addition to the galactic cosmic rays, the anomalous cosmic rays, which are quite variable.

These cosmic ray particles present a constant source of background. They are so energetic that they cannot be effectively shielded in an instrument on a spacecraft, since the necessary mass would be prohibitive. For both IBEX-Lo and IBEX-Hi, the particle detectors are deep inside the sensor, which gives some shielding. However, the best reduction of this background is the use of a triple coincidence technique for the particle detection, together with TOF identification (IBEX-Lo) or TOF gates (IBEX-Hi).

During quiescent periods, measurements from the X-ray satellite Chandra show that cosmic rays produce a count rate of about $1.6 \mathrm{~s}^{-1} \mathrm{~cm}^{-2}$ in the MCP detector (Juda et al. 2003). This results in a count rate of $40 \mathrm{~s}^{-1}$ for each of the four IBEX-Lo MCP detectors. Using a TOF window of $200 \mathrm{~ns}$ this gives a double rate of $3.2 \cdot 10^{-4} \mathrm{~s}^{-1}$ and a triple rate of $2.6 \cdot 10^{-9} \mathrm{~s}^{-1}$. With the false ion detections randomly distributed in the mass spectra their separation from the signal will be feasible.

Measurements from the Genesis Ion Monitor using energy channels significantly above the solar wind energy show total CEM background count rates of approximately $0.5 \mathrm{~s}^{-1}$ (Steinberg 2007). Similarly, we anticipate that the IBEX-Hi CEM count rate from penetrating radiation will be $0.5 \mathrm{~s}^{-1}$. Using a TOF window of $100 \mathrm{~ns}$ (corresponding to the long coincidence window) this gives a double rate of $2.5 \cdot 10^{-8} \mathrm{~s}^{-1}$ and a triple rate of approximately $1.2 \cdot 10^{-15} \mathrm{~s}^{-1}$. Although these estimated background rates are very low, they will add directly to the signal rates since there is no mass analysis (i.e., TOF analysis) in IBEX-Hi. To minimize the background from penetrating radiation in the IBEX-Hi sensor, the CEMs are positioned such that no straight penetrating particle trajectory can go through all three CEMs. Thus, a triple coincidence is limited to penetrating particles actually crossing the two carbon foils. Backgrounds due to penetrating radiation are discussed in more detail in Sect. 3.1.2.

Solar Energetic Particle (SEP) events are another source of penetrating radiation. However, their total duration of events lasts less than $2 \%$ of the mission. Data during this $2 \%$ of the mission are removed from analysis. Solar Energetic Particle events are easily identified for removal by the increase in non-coincident rates of all detectors, and these rates are independent of energy passband and incident angle. Data from other spacecraft also provide the arrival time and duration of these SEP events. 


\subsection{Neutral Atom Background}

Competing ENA signals for the IBEX objective originate from the terrestrial ring current and plasma sheet, which we will refer to as foreground. Heliospheric and interstellar neutral fluxes are relatively low, so care must be taken to identify look directions where the terrestrial ENA intensities dominate. For comparison, typical strong storm-time neutral fluxes from the Earth's magnetosphere (ring current) in the energy range of $1000-2000 \mathrm{eV}$ are about $4 \cdot 10^{4} \mathrm{ENAs} /\left(\mathrm{cm}^{2} \mathrm{~s} \mathrm{sr}\right)$, which exceeds even the highest predictions of ENAs from the termination shock region by a factor of 10 (see Fig. 1). ENAs from high latitude ionospheric outflow at energies around $50 \mathrm{eV}$ range from $6 \cdot 10^{4}$ to $1 \cdot 10^{6} \mathrm{ENAs} /\left(\mathrm{cm}^{2} \mathrm{~s} \mathrm{sr}\right)$. These neutral fluxes can vary significantly on timescales of tens of minutes.

\subsubsection{Ring current}

To estimate the extent of terrestrial ENA intensities we simulate the ENA intensities from the expected apogee $\left(40 R_{E}\right)$ and the minimum science operations altitude $\left(10 R_{E}\right)$. Our goal is to provide upper limits on the expected background. Most of the time the terrestrial ENA background will be orders of magnitude lower than the upper limits given here. Energetic neutral atom simulations are produced from runs of the Comprehensive Ring Current Model (CRCM) (Fok et al. 2001, 2003). The boundary conditions of this model run were taken from an event with an unusually dense plasma sheet leading to a strong ring current. Only protons have been assumed since the $\mathrm{O}^{+}$intensities (not energy densities!) and oxygen ENA intensities are often less than those of protons. Typical storm-time, ring-current ion spectra have been compiled by Kistler et al. (1989), and the ring current and plasma sheet ion distribution have been reported by several authors (Hamilton et al. 1988).

Estimated ring-current-hydrogen ENA intensities are shown in Fig. 9 together with skymaps of the ENA emissions from the vantage points $y=40 R_{E}$ (panel a) and $y=10 R_{E}$ (panel b). These simulated images have a high angular resolution and are meant to be used as reference ENA distributions representing the ENA foreground outside the instrument. The line plot (solid lines in panel c) in Fig. 9 shows the ENA intensity in the sensor's $7^{\circ}$-wide FOV as a function of look angle for various vantage points. The two strong peaks visible near zero degree spin angle result from the low-altitude emission, which is produced by the charge exchange between the magnetospheric ions and the upper atmosphere. To correctly model the low-altitude emission, multiple charge exchange and charge stripping interactions must be taken into account. In the present simulation we have approximated the low-altitude interaction by taking a "hard" shell at $350 \mathrm{~km}$ altitude below which we assume all ions to be lost to the atmosphere. This is acceptable for the purpose of this paper, since it is already implemented in the science operations that the IBEX sensors will not take any measurements when inside 10 Earth radii (McComas et al. 2009, this issue).

It is important to note that the ENA emissions from the plasma sheet are clearly visible in both IMAGE/HENA (Brandt et al. 2002) and IMAGE/MENA (McComas et al. 2002) measurements. Therefore, they probably represent the most extended foreground signal relevant to the IBEX observations. From its near-equatorial orbit it is anticipated that IBEX may detect ENA emissions from the plasma sheet as far down the tail as $x=-20 R_{E}$ or more, in the Earthward hemisphere. These ENA emissions are easily avoided by excluding the Earthward hemisphere when IBEX is outside the plasma sheet region. In fact, the vertical scans of the plasma sheet present a very valuable diagnostic tool for the plasma sheet itself. 
(a)

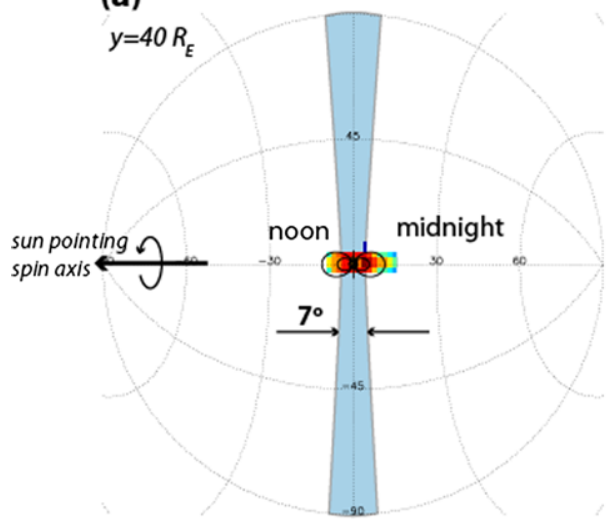

(b)

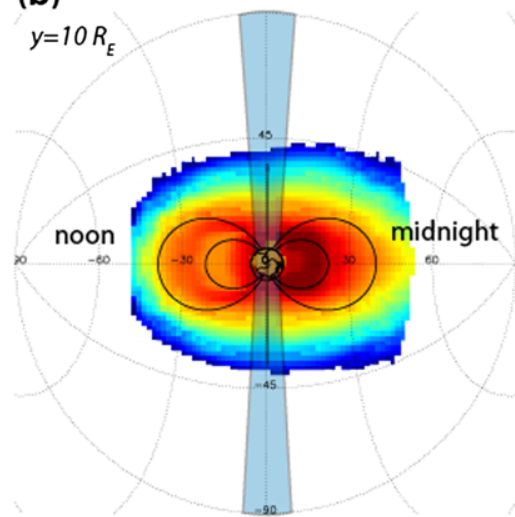

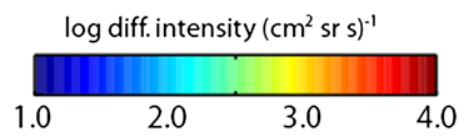

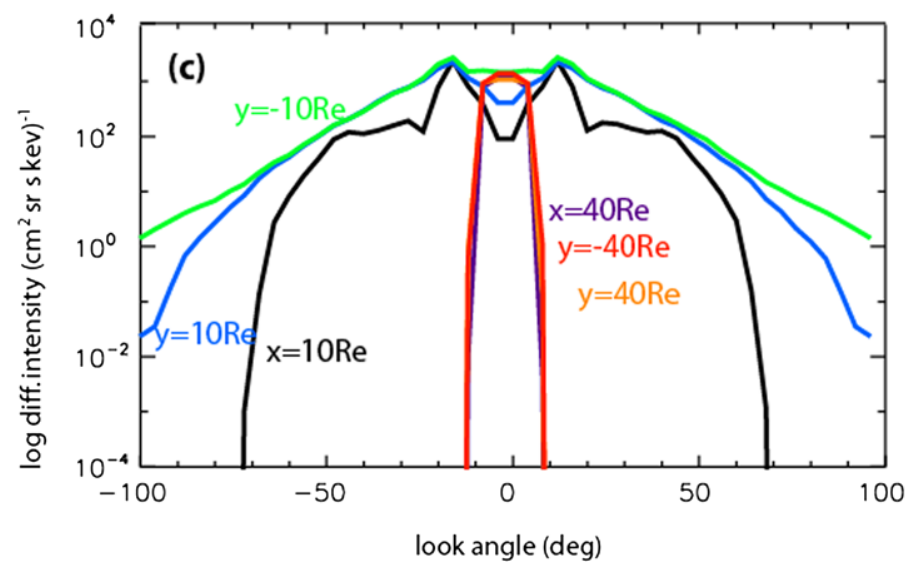

Fig. 9 Simulated worst-case terrestrial ENA intensities in the 1-6 keV range for the ring current and the plasma sheet. (a) Full sky map of ENA emissions taken from $y=40 R_{E}$. The blue swath represents the angular width of the IBEX telescope. (b) Same as (a) but from $y=10 R_{E}$. (c) Lines represent the ENA intensities measured by a spinning $7^{\circ}$ wide telescope from various vantage points as indicated

\subsubsection{IMAGE/HENA Backgrounds}

Even though the IMAGE/HENA instrument was designed to image magnetospheric ENAs (Mitchell et al. 2000), it scanned almost the entire sky like the two IBEX sensors. So there were portions of the scans that could be used to search for ENA sources beyond the heliospheric termination shock. No clear sources of heliospheric emission were detected above the various background sources. There were three significant sources of background in the HENA data, and they were carefully quantified as part of the search for heliospheric sources: 1) ultraviolet radiation (of solar, terrestrial, and heliospheric origin) falling on the front foil, 2) penetrating particles and particles passing through the electrostatic deflection system of 
the collimators and 3) ENAs from magnetospheric sources. Note that these background sources were not significant for the imaging of magnetospheric ENAs, which have intensities several orders of magnitude larger than the expected intensities of ENAs from the heliosheath.

The coincidence logic for HENA required a pulse on a back foil following a pulse on the front foil within a set of TOF windows. There was a finite count rate on both the front and back foils. The counts on the front foil were primarily due to ultraviolet radiation, and they tended to change with solar activity. The counts on the back foil generally remained nearly constant and are attributed to cosmic rays penetrating the instrument. The accidental coincidence rates estimated from the measured start (front foil) and stop (back foil) counts within the TOF windows were comparable to (but less than) the actual total rates. There were also intermittent periods when there were enhanced background count rates that were closely correlated to increases in solar energetic protons measured by the ACE/EPAM instrument. These periods were easy to identify and eliminate from the data set. Finally, very conservative geometric criteria (based on the geometry of geomagnetic field lines in the particle trapping regions) were set to separate ENAs produced in the magnetosphere from those produced in the heliosheath. In the end, IMAGE/HENA did detect regions of the sky where the actual counts (surviving the culling based on the selection criteria) exceeded the estimated accidental counts. Although it could be a candidate source of ENAs from the heliosheath, it is better ordered in the sky in GSE (Sun-Earth centered) coordinates than it is in ECI (inertial ecliptic) coordinates, thus suggesting a residual magnetospheric contribution from the direction of the geotail.

\subsubsection{Neutral Solar Wind}

Neutral solar wind (NSW) originates from solar wind ions, which become neutralised during their travel from the Sun through interplanetary space. Consequently, NSW moves in the anti-Sunward direction with solar wind velocity, i.e., $300-800 \mathrm{~km} \mathrm{~s}^{-1}$. Observational evidence of NSW thus far is very scarce because of the difficulty in measuring neutral particle fluxes several orders of magnitude lower than the solar wind flux, while simultaneously being exposed to full sunlight. The only observation thus far was made with the Low Energy Neutral Atom (LENA) imager on IMAGE, from which a NSW fraction of about $10^{-4}$ was derived (Collier et al. 2001). Enhancements in the NSW flux due to charge exchange in the geocorona were observed, with the NSW fraction increasing to a few times $10^{-4}$ (Collier et al. 2001). Since NSW flow direction is roughly perpendicular to the FOVs of the sensors it is of little concern for the background discussion.

A special case of the neutral solar wind is produced just upstream of the subsolar region of the magnetopause. The solar wind slows and is heated across the bow shock and as it approaches the magnetopause. The hot $(\sim 1 \mathrm{keV}$ thermal energy), slow (several 10 to $\sim 100 \mathrm{~km} / \mathrm{s}$ ) solar wind is charge exchanged by the Earth's geocorona in the region. Like the ring current and plasma sheet neutrals, this background will be removed from the ENA data when they are in the FOV.

\subsubsection{Pre-termination Shock ENAs}

Proton distributions in the inner heliosheath are a function of the distance to the termination shock (TS) since pickup ions increase the random thermal energy of the solar wind and are more abundant for larger TS distances. These protons can be accelerated (e.g., at co-rotating interaction regions) and form suprathermal tails on the solar wind and pickup ion distributions. Goeckler (2003) has summarized the behaviour of the ubiquitous suprathermal tails. 
They are characterised by power-laws in velocity space in the solar wind frame. When these ions have charge-exchange collisions with the interstellar neutral atoms that flow through the heliosphere, ENAs will be generated, which may be observed in Earth orbit. These ENAs are created inside the TS, thus they are called pre-TS ENAs and will interfere with the heliospheric ENAs of interest. However, it has been concluded earlier (McComas et al. 2004) that these pre-TS ENAs generated from accelerated protons inside the TS are not a significant background.

\subsubsection{Summary}

In summary, the strongest terrestrial ENA signal comes from the ring current, but the most extended consists of the ENA emissions coming from the plasma sheet during storms. Therefore, to be conservative, any scans across the Earthward hemisphere should be treated with extreme caution even at down tail distances at $x=-20 R_{E}$. Similarly, any scans at $x=10$ $R_{E}$ should be treated with extreme caution. However, such scans will also present valuable opportunities for investigating global plasma sheet and subsolar magnetosheath properties.

\section{Internal Backgrounds}

Internally generated signals that compete with the expected ENA observations include all sources that randomly trigger the detectors without external stimulus, including the generation of ions internally through knock-off from grids in the particle path. Some additional background sources for IBEX-Lo and IBEX-Hi were identified during the extensive testing and calibration of the sensors and are discussed in the following subsections.

\subsection{Random Background Sources}

Random MCP or CEM pulses arising from radionuclide decay (mostly from the ${ }^{40} \mathrm{~K}$ decay) fall into this category. Typical rates are for MCPs in the range of 0.1-1 counts per second per $\mathrm{cm}^{2}$. The resulting background is lower than what is expected from penetrating cosmic rays.

Also electronic noise on the signal lines may appear as valid particle detection in a channel. These events occur in a random fashion, with a finite chance for a double coincidence, and a smaller chance for a triple coincidence, which contributes to the background in the particle identification.

Other sources of background come from the high voltage power supplies needed for the ion optical system of each sensor. The generation of high voltages employs oscillators, which may contribute electronic noise on the signal lines if not shielded carefully. Moreover, tiny discharges below the detection level of the partial discharge measurements may create an ion signal in the detector. The typical detection limit of partial discharge measurements is at a charge level of $\mathrm{pC}$, which means a discharge involving $10^{6}$ electrons can happen unnoticed. If some of the electrons propagate to the detector, or create ions on their way (by electron impact ionisation or electron stimulated desorption) such a discharge will contribute to the background. 

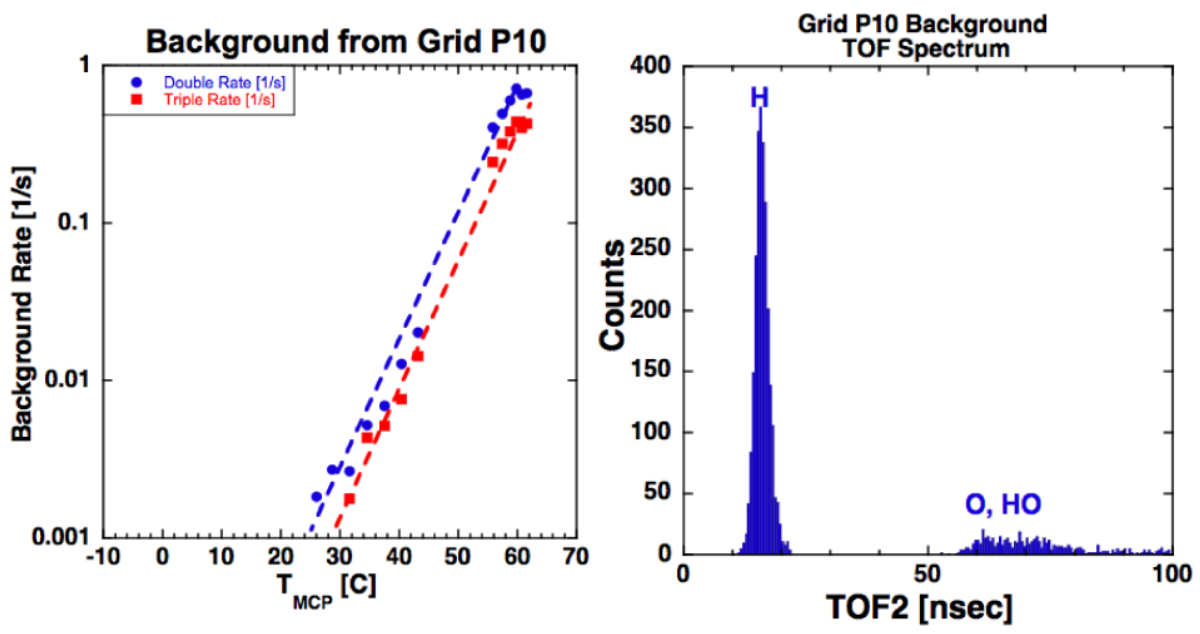

Fig. 10 Internal background of IBEX-Lo during thermal test. Left: Background rate as a function of temperature. Right: Mass spectrum of background signal

\subsubsection{IBEX-Lo Internal Background}

To identify any possible internal background sources, IBEX-Lo was operated long term in a mode that prevented any entry of external particles during thermal vacuum testing. No outside stimulus was active, e.g. there was no neutral particle beam. Also, all but one of the ion gauges of the vacuum system were turned off to minimise ambient ions and electrons. The operating ion gauge was of Penning-type and was baffled to largely suppress ion and electron emission into the test chamber. Figure 10 shows a result of the background determination performed during thermal vacuum test, where events with valid TOF combinations for $\mathrm{H}, \mathrm{O}$, and $\mathrm{OH}$ were indeed observed. The observed background is strongly temperature dependent (Fig. 10, left panel), which indicates that this background involves outgassing (most likely water) at least in an indirect manner. Figure 10, right panel shows a mass spectrum of this background, which is dominated by hydrogen, a fragment from water. The expected operating temperature of IBEX-Lo in space is in the range from $0^{\circ} \mathrm{C}$ to $+18^{\circ} \mathrm{C}$, which results in background rates of $2.6 \cdot 10^{-4} \mathrm{~s}^{-1}$ and $1.25 \cdot 10^{-4} \mathrm{~s}^{-1}$, for double and triple coincidence detection, respectively, at the upper end of the expected temperature range. When calculating back to the equivalent flux on the conversion surface, this internal background corresponds to about $0.5 \mathrm{~s}^{-1}$ hydrogen ENAs arriving on the conversion surface.

Remember, this background is observed without any external stimulus. Inspection of background location on the four azimuth quadrants in the TOF unit showed that the most likely origin of these ions is the last electrode of the ESA subsystem, P10 (located just before the post-acceleration path to the TOF section), because this background is mostly observed in one quadrant. Figure 11, left panel, shows a schematic view of the ion-optical system near the post-acceleration region. As the first step in the creation of this background, electrons are emitted from the grids of electrode P10, probably via field emission from the edges of the grid wires (red arrow in Fig. 11). The electrons are accelerated toward the TOF unit and impinge the TOF structures with $10 \mathrm{keV}$ and more. If a positive ion is released as a result of the electron impact, this positive ion will be accelerated toward electrode P10 (black arrow in Fig. 11). Upon impact on the grid and other structures there is the possibility that a negative ion is sputtered that in turn is accelerated by the post-acceleration potential toward the TOF 


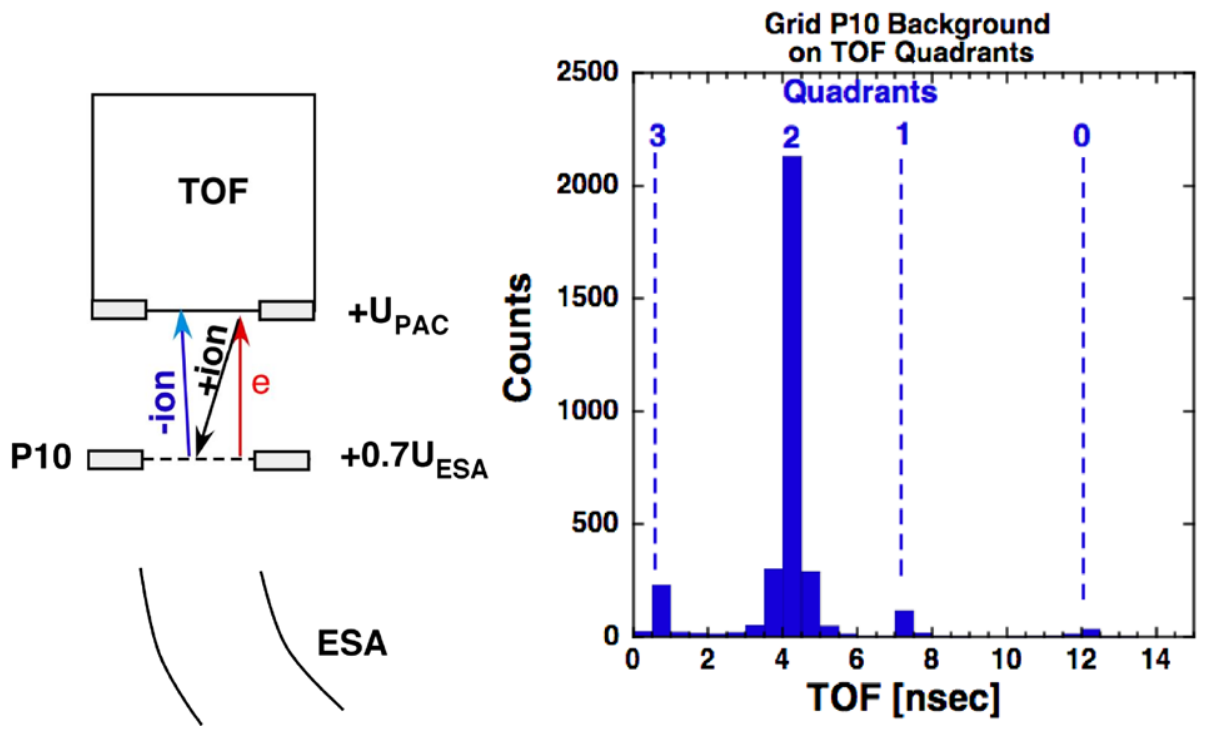

Fig. 11 Left: Schematic representation of the ion optical system near the post-acceleration region. Right: Histogram of the azimuth sector of background ions

(blue arrow in Fig. 11). If the ion passes the carbon foil it will be detected. An energetic electron impinging onto a metal surface can only remove adsorbates, but not sputter, and apparently is temperature dependent. Therefore, upon better evacuation of the sensor during the mission this background is expected to become smaller. Further support for this concept of background generation arises from the measurement of the azimuth angle of the registered ion in the TOF unit (see Fuselier et al. 2009, this issue, for a detailed sensor description). The registered background ions are mostly clustered in one azimuth quadrant, as can been seen in Fig. 11, right panel. Such a narrow distribution in azimuth can only happen if they are created after ESA, close to the TOF unit. Ions originating on the conversion surface will spread into at least two azimuth sectors even if they start out at a point source on the conversion surface (Wieser et al. 2007; Fuselier et al. 2009, this issue).

This observation also presents an opportunity to further reduce the background in the data analysis. Events from the MCP quadrant with the highest internal background rate can be culled out because the MCP quadrant information is retained in the event data.

\subsubsection{IBEX-Hi Internal Background}

Internal backgrounds in IBEX-Hi were measured during extended periods of operation when the sensor was fully operational but without external stimuli, i.e., no incident ion or ENA beam.

As a first test, the IBEX-Hi detector subsystem was continuously operated for about $26 \mathrm{~h}$ for the determination of internal backgrounds. The background rate of a single CEM detector was in the range $(4.0-8.6) \cdot 10^{-2} \mathrm{~s}^{-1}$. For the double rates, i.e., the registration of a signal in two CEM detectors within a short time interval, we obtained rates of about 1.6 $10^{-2} \mathrm{~s}^{-1}$. For the individual coincidence combinations of CEM detectors the background rates were as follows: $5.4 \cdot 10^{-3} \mathrm{~s}^{-1}$ for coincidences between detectors $\mathrm{A}$ and $\mathrm{B}$; $9.2 \cdot 10^{-3} \mathrm{~s}^{-1}$ for the BC coincidences; and $1.0 \cdot 10^{-3} \mathrm{~s}^{-1}$ for the AC coincidences. The 
Table 3 IBEX-Hi average background count rates for exclusive (non-coincident) single events and a subset of coincidence types measured over 19.6 hours of quiescent operation during cross calibration

\begin{tabular}{lll}
\hline Measurement & & Background rate [cnt/s] \\
\hline Singles & CEM A & $89 \cdot 10^{-3}$ \\
& CEM B & $115 \cdot 10^{-3}$ \\
& CEM C & $69 \cdot 10^{-3}$ \\
& CEM D & $12 \cdot 10^{-3}$ \\
Double coincidences & Long AB & $2.8 \cdot 10^{-3}$ \\
& Long BC & $4.2 \cdot 10^{-3}$ \\
& Qual(Not_C) AC & $0.13 \cdot 10^{-3}$ \\
Triple coincidences & Qual(Not_C) ABC & $0.58 \cdot 10^{-3}$ \\
& Long ABC & $1.04 \cdot 10^{-3}$ \\
\hline
\end{tabular}

background rate for triple coincidence $(\mathrm{ABC})$ events were $2.9 \cdot 10^{-3} \mathrm{~s}^{-1}$. The background measurement for the single events, the double coincidence rates, and the triple coincidence rate is shown in Fig. 12.

For the full IBEX-Hi sensor the average background count rates were measured over 19.6 hours during the IBEX sensor cross calibration campaign (IBEX-Hi Cal 4 campaign), and the results are listed in Table 3. The singles count rates in each CEM detector were $<0.2 \mathrm{~s}^{-1}$. While the coincident count rates are higher than expected based on random coincidence of the background singles rates in the detectors, we have found that ambient gamma rays are associated with a majority of the background coincidence events observed in the detector subsystem.

The IBaM was also continuously operated for $19.1 \mathrm{~h}$ during cross-calibration to determine its internal background. Its background rate was found to be $1.15 \cdot 10^{-2} \mathrm{~s}^{-1}$ (see panel "D" in Fig. 12 for the actual measurement).

During tests with the engineering model (EM) the sensitivity of the IBEX-Hi detector subsystem to gamma rays was noticed. We used laboratory TOF electronics to measure the TOF between detected events in all coincidence combinations. The EM detector subsystem measures coincidence events from ambient background, likely to be $\gamma$-rays from the surrounding building concrete because of accidental coincidences. Possible $\gamma$-ray emitters in concrete are ${ }^{40} \mathrm{~K},{ }^{232} \mathrm{Th}$ and daughters, and ${ }^{235 / 238} \mathrm{U}$ and daughters. We subsequently measured the background $\gamma$-ray environment in the LANL calibration facilities, showing a background flux $6.3 \gamma \mathrm{cm}^{-2} \mathrm{~s}^{-1}$ between $0.2-3 \mathrm{MeV}$. Analysis of the measured background $\gamma$-ray spectrum indicated that ${ }^{40} \mathrm{~K}$ and ${ }^{226} \mathrm{Ra}$ were the dominant $\gamma$-ray line emitters on top of a broad $\gamma$-ray continuum that increased with decreasing energy as shown in Fig. 13.

Figure 14 shows a comparison of TOF histograms using laboratory TOF electronics for an incident $3.5 \mathrm{keV}$ proton beam (top panel), a background measurement (middle panel) and a background measurement with an additional ${ }^{137} \mathrm{Cs} \gamma$-ray source placed next to the sensor (bottom panel). The left, centre, and right histograms for each of these cases represent triple coincidences in which the first event was recorded in CEMs A, B, and C, respectively. The background run spanning $2.3 \cdot 10^{5} \mathrm{sec}$ (during which the triple coincidence rate was $6 \cdot 10^{-3}$ $\mathrm{s}^{-1}$ ) and the run with the ${ }^{137} \mathrm{Cs}$ source over $2.5 \cdot 10^{4} \mathrm{sec}$ are very similar. In particular, for both runs the number of triple coincidences in which CEM C was the first detector to register an event is comparable to the number of triple coincidences in which CEM A was the first to register a pulse. In contrast, the proton beam never caused a triple coincidence in which CEM 


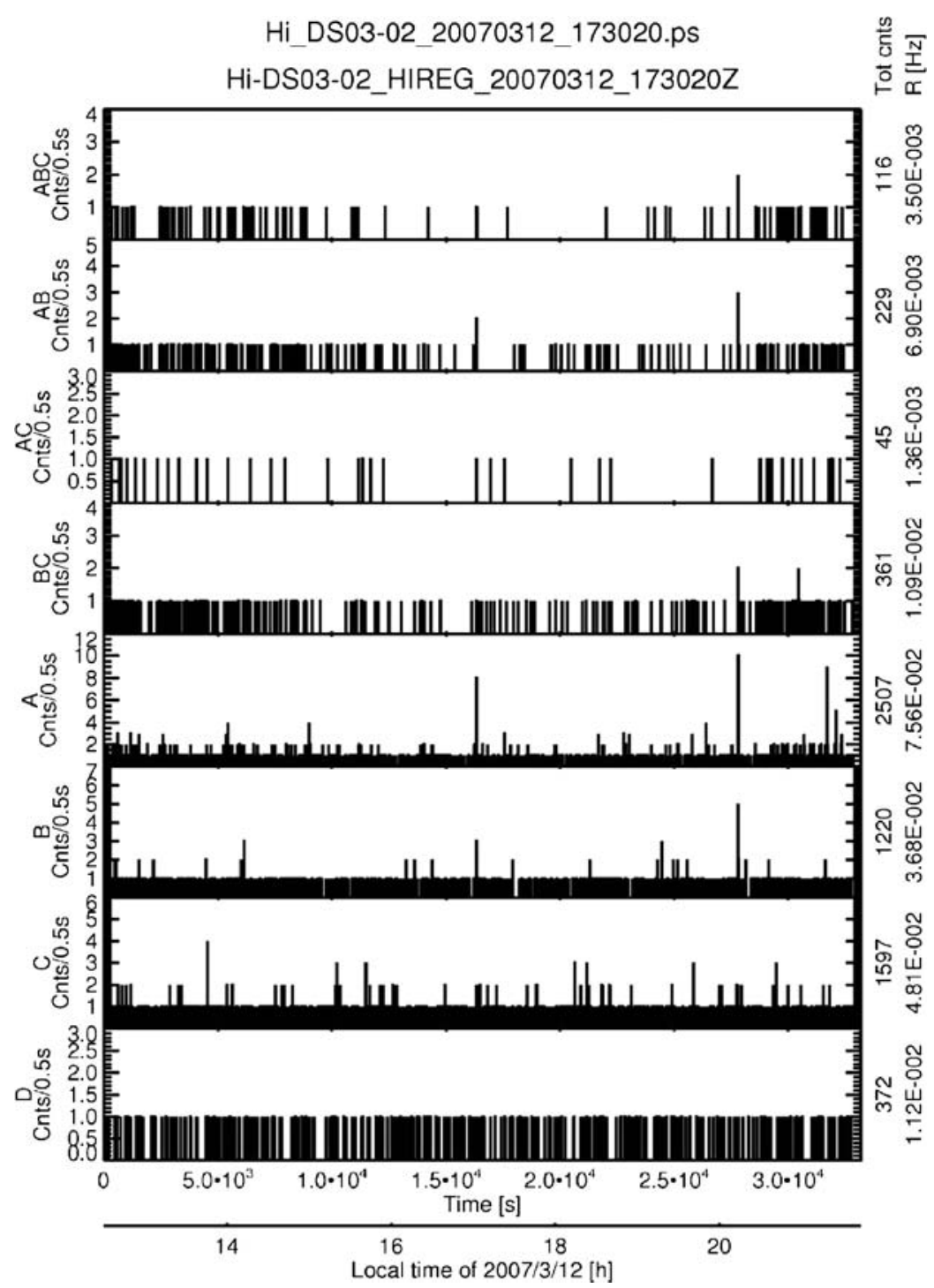

Fig. 12 Background measurement of the IBEX-Hi detector section, showing the single events, the double rates, and the triple rate

$\mathrm{C}$ registered the first pulse, which is the direct result of $\mathrm{H}$ sequentially transiting detector chambers A, B, and finally $\mathrm{C}$. We can therefore conclude that the measured background during testing in the LANL facility is likely the result of the penetrating $\gamma$-ray radiation with its spectrum shown in Fig. 13. Also, the IBaM showed some susceptibility to $\gamma$-rays; its background rate went up to $1.22 \cdot 10^{-1} \mathrm{~s}^{-1}$ when exposed to $\gamma$-rays.

We note that ${ }^{137} \mathrm{Cs}$ also emits $1.175 \mathrm{MeV}$ electrons that have a range of about $2.3 \mathrm{~mm}$ in Aluminium, but these electrons were completely blocked by the $\mathrm{Al}$ walls of minimum thickness $>2.5 \mathrm{~mm}$ surrounding the interior of the detector chambers and CEM detectors. 
Fig. $13 \gamma$-ray spectrum as measured at the LANL calibration facility

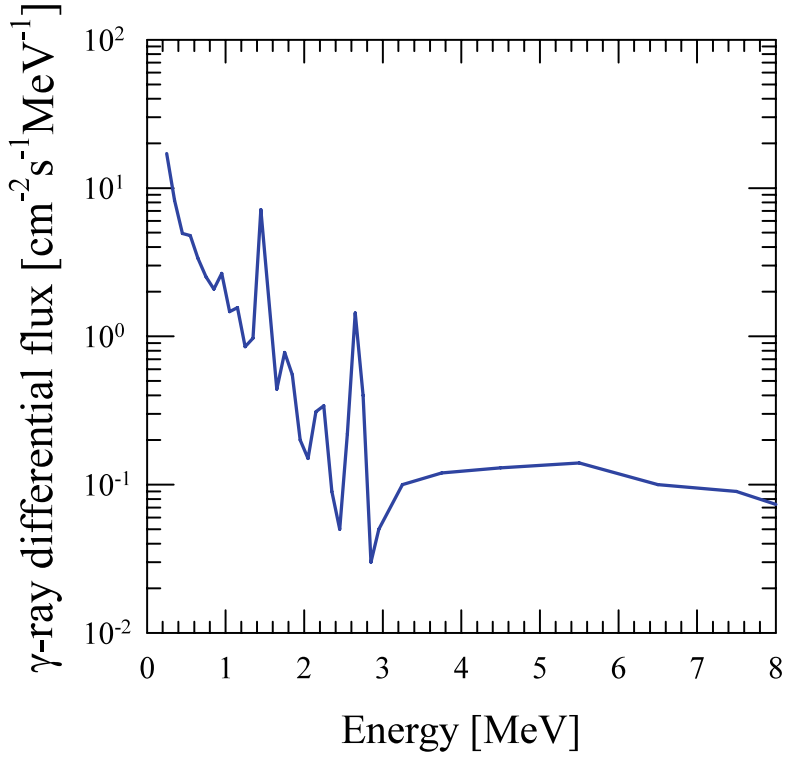

A first

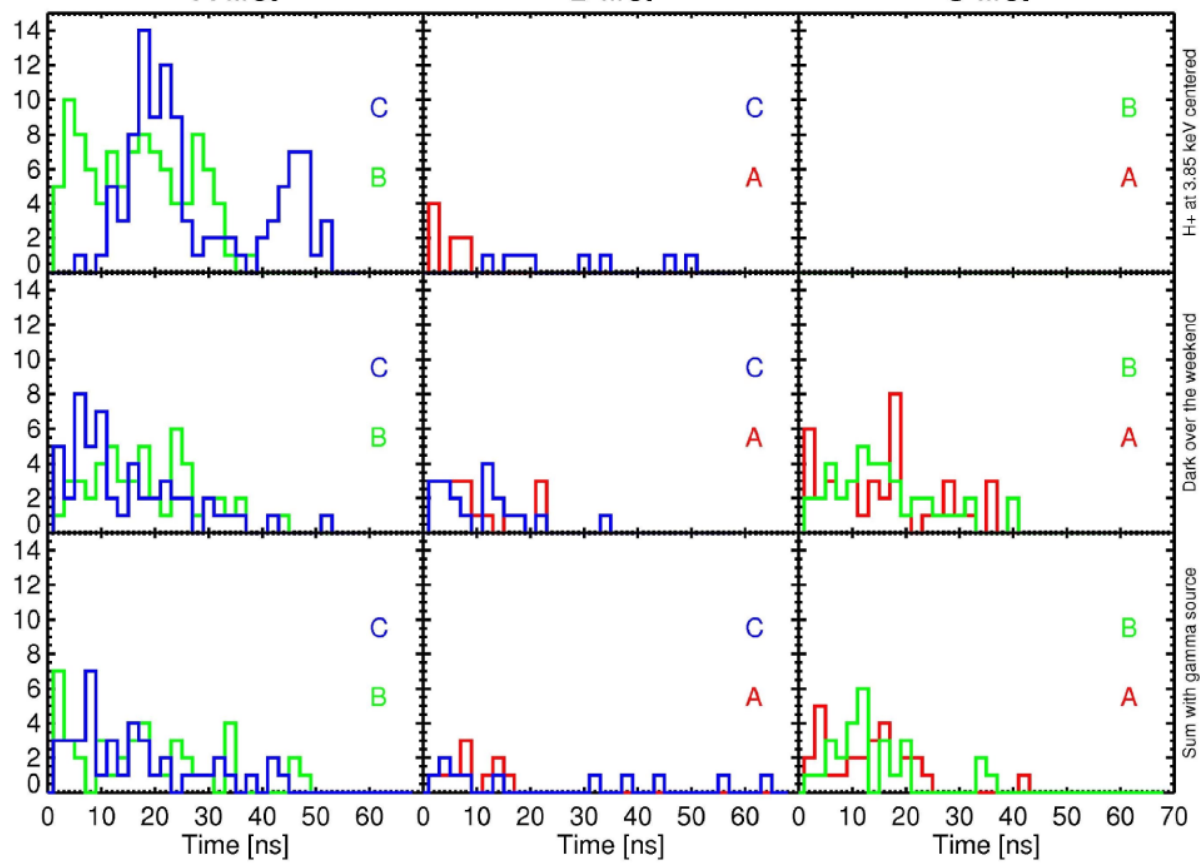

Fig. 14 Triple coincidence TOF distributions for a $3.85 \mathrm{keV}$ hydrogen beam (top), a background ("Dark" run) distribution, and a background distribution with a ${ }^{137} \mathrm{Cs} \gamma$-ray source was placed next to the sensor 
The majority of coincident counts listed in Table 3 are a result of the laboratory $\gamma$-ray environment. From the estimates of cosmic ray induced background presented in Sect. 2.4 we expect that in space the measured background count rates are significantly lower, and the present numbers represent overestimates of the background rates. In any case, the triple coincidence count rate in which CEM C generates the first pulse will directly monitor the presence and intensity of penetrating radiation in IBEX-Hi. From the laboratory measurements (see Fig. 14), we know how to relate the background count rate of C-first to the background count rates A-first and B-first, therefore we can subtract the contribution of penetrating radiation in the latter two rates in the data analysis.

\section{Conclusions}

The IBEX sensors were each designed to maximize the signal-to-noise ratio by utilising a large geometric factor, single-pixel camera design with extensive background rejection. Natural background sources in the IBEX sensors include ENAs from the Earth's magnetosphere and magnetosheath, which actually represent a localised foreground for the planned heliospheric observations. Intensities of these sources are much larger than the ENA signal from the outer heliosphere. This natural foreground will be culled from the data, and the high altitude of the IBEX orbit helps reduce the effect of this foreground on the overall sky maps. Energetic neutral atoms can also be produced in the immediate vicinity of the spacecraft by charge exchange of the solar wind or magnetosheath with the outgassing products (mostly water) from the spacecraft. These backgrounds have been estimated and are found to be orders of magnitude lower than the expected signal. Of course, the outgassing from the spacecraft and the sensors have been minimised by stringent cleanliness control.

Energetic ions and photons interact with gas inside two regions in the IBEX collimators to produce ions that can masquerade as ENAs. This background source represents the most complex and highest background in the sensors. Background from Lyman- $\alpha$ was measured in the sensors during calibration and a background level was determined. Finally, additional background specific to the sensors includes negative ion production deep inside the sensor (IBEX-Lo) and sensitivity of the detector system to penetrating radiation.

The background sources for the IBEX mission have been extensively investigated through simulation, analysis using up-to-date information from other missions, and extensive testing during sensor calibration. Based on this extensive investigation, we conclude that there is ample signal-to-noise margin for measurements over the energy range of the IBEX mission.

\section{References}

F. Allegrini, G.B. Crew, D. Demkee, H.O. Funsten, D.J. McComas, B. Randol, B. Rodriguez, N.A. Schwadron, P. Valek, S. Weidner, The IBEX-Hi background monitor, Space Sci. Rev. (2009, this issue)

D.J. Baker, G.J. Romick, The Raleigh: Interpretation of the unit in terms of column emission rate or apparent radiance expressed in SI units. Appl. Optics 15, 1966-1968 (1976)

J. Berkowitz, Atomic and Molecular Photoabsorption (Academic Press, San Diego, 2002)

J.L. Bertaux, E. Kyrölä, E. Quémerais, R. Pellinen, R. Lallement, W. Schmidt, M. Berthé, E. Dimarellis, J.P. Goutail, C. Taulemesse, C. Bernard, G. Leppelmeier, T. Summanen, H. Hannula, H. Huomo, V. Kehlä, S. Korpela, K. KLeppälä, E. Strömmer, J. Torsti, K. Viherkanto, J.F. Hochedez, G. Chretiennot, R. Peyroux, T. Holzer, SWAN: A study of solar wind anisotropies on SOHO with Lyman alpha sky mapping. Sol. Phys. 162, 403-439 (1995) 
P.C. Brandt, R. DeMajistre, E.C. Roelof, D.G. Mitchell, S. Mende, IMAGE/HENA: Global ENA imaging of the plasmasheet and ring current during substorms. J. Geophys. Res. 107, 1454 (2002). doi:10.1029/2002JA009307

S.V. Chalov, H.J. Fahr, V.V. Izmodenov, Evolution of pickup proton spectra in the inner heliosheath and their diagnostics by energetic neutral atom fluxes. J. Geophys. Res. 108(A6), 1266 (2003). doi:10.1029/2002JA009492

M.R. Collier, T.E. Moore, K.W. Ogilvie, J.D. Chornay, J.W. Keller, S. Boardsen, J.L. Burch, B. El Marji, M.-C. Fok, S.A. Fuselier, A.G. Ghielmetti, B.L. Giles, D.C. Hamilton, B.L. Peko, J.M. Quinn, T.M. Stephen, G.R. Wilson, P. Wurz, Observations of neutral atoms from the solar wind. J. Geophys. Res. 106, 24893-24906 (2001)

H.-J. Fahr, K. Scherer, Energetic neutral atom fluxes from the heliosheath varying with the activity phase of the solar cycle. ASTRA Astrophys. Space Sci. Trans. 1, 3-15 (2004)

M.-C. Fok, R.A. Wolf, R.W. Spiro, T.E. Moore, Comprehensive computational model of Earth's ring current. J. Geophys. Res. 106, 8417-8424 (2001)

M.-C. Fok, T.E. Moore, G.R. Wilson, J.D. Perez, X.X. Zhang, P.C. son Brandt, D.G. Mitchell, E.C. Roelof, J.M. Jahn, C.J. Pollock, R.A. Wolf, Global ENA image simulations. Space Sci. Rev. 109, 77-103 (2003)

C. Fröhlich, J. Lean, Solar radiative output and its variability: evidence and mechanisms. Astron. Astrophys. 12, 273-320 (2004)

H.O. Funsten, A.A. Guthrie, R.W. Harper, K.H. Kihara, M.P. Manzo, M.J. Fagan, D.J. McComas, S. Weidner, F. Allegrini, D. Everett, B. Rodriguez, G. Dunn, S. Pope, J. Hanley, P. Valek, E. Moebius, J. Nolin, S. Ellis, D. Heirtzler, B. King, H. Kucharek, S. Turco, S. Zaffke, D. Reisenfeld, P. Janzen, S.A. Fuselier, M. Gruntman, E. Roelof, P. Wurz, D. Piazza, L. Saul, P. Bochsler, The interstellar boundary explorer high energy (IBEX-Hi) neutral atom imager. Space Sci. Rev. (2009, this issue)

S.A. Fuselier, A.G. Ghielmetti, E. Hertzberg, A.S. Moore, D. Isaac, J.W. Hamilton, C. Tillier, E. Moebius, M.S. Granoff, D. Heirtzler, B. King, H. Kucharek, S. Longworth, J. Nolin, S. Turco, S. Ellis, J. Googins, F. Kudirka, J. Tyler, M. Vosbury, G. Clark, M. O’Neal, P. Wurz, J.A. Scheer, L.A. Saul, D. Piazza, P. Bochsler, M. Wieser, C. Schlemm, D.J. McComas, J. Scherrer, S. Pope, H.O. Funsten, D. Chornay, J. Lobell, T.E. Moore, P. Rosmarynowski, T. Friedmann, R.J. Nemanich, The IBEX-Lo Sensor. Space Sci. Rev. (2009, this issue)

S. Graf, K. Altwegg, H. Balsiger, P. Bochsler, B. Fiethe, E. Montagnon, Thruster plumes—a source for high pressure and contamination at the payload location. J. Spacecr. Rockets 45, 57-64 (2008)

A. Galli, P. Wurz, H. Lammer, H.I.M. Lichtenegger, R. Lundin, S. Barabash, A. Grigoriev, M. Holmström, H. Gunell, The hydrogen exospheric density profile measured with ASPERA-3/NPD. Space Sci. Rev. 126, 447-467 (2006)

G. Goeckler, Ubiquitous suprathermal tails on the solar wind and pickup ion distributions, in Proc. Solar Wind X, Am. Inst. Phys., CP679 (2003), pp. 583-588

J.B. Greenwood, I.A. Chutjian, S.J. Smith, Measurements of the absolute, single charge-echange cross section of $\mathrm{H}^{+}, \mathrm{He}^{+}$, and $\mathrm{He}^{2+}$ with $\mathrm{H}_{2} \mathrm{O}$ and $\mathrm{CO}_{2}$. Astrophys. J. 529, 605-609 (2000)

M. Gruntman, E.C. Roelof, D.G. Mitchell, H.J. Fahr, H.O. Funsten, D.J. McComas, Energetic neutral atom imaging of the heliospheric boundary region. J. Geophys. Res. 106, 15767-15782 (2001)

D.C. Hamilton, G. Gloeckler, F.M. Ipavich, W. Stüdemann, B. Wilken, G. Kremser, Ring current development during the great geomagnetic storm of February 1986. J. Geophys. Res. 93, 14343-14355 (1988)

J. Heerikhuisen, N.V. Pogorelov, G.P. Zank, V. Florinski, The effects of global heliospheric asymmetries on energetic neutral atom sky maps. Astrophys. J. 655, L53-L56 (2007)

M. Hilchenbach, K.C. Hsieh, D. Hovestadt, B. Klecker, H. Grünwaldt, P. Bochsler, F.M. Ipavich, F. Gliem, W.I. Axford, H. Balsiger, W. Bornemann, A. Bürgi, M.A. Coplan, A.B. Galvin, J. Geiss, G. Gloeckler, S. Hefti, D.L. Judge, R. Kallenbach, P. Laeverenz, M.A. Lee, S. Livi, G.G. Managadze, E. Marsch, E. Möbius, M. Neugebauer, H.S. Ogawa, K.U. Reiche, M. Scholer, M.I. Verigin, B. Wilken, P. Wurz, Detection of 55-80 keV hydrogen atoms of heliospheric origin by CELIAS/HSTOF on SOHO. Astrophys. J. 503, 916-921 (1998)

R.R. Hodges, E.L. Breig, Ionosphere-Exosphere coupling through charge exchange and momentum transfer in hydrogen-proton collisions. J. Geophys. Res. 96, 7697-7708 (1991)

K.C. Hsieh, P.C. Frisch, J. Giacalone, J.R. Jokipii, J. Kota, D.E. Larson, R.P. Lin, J.G. Luhmann, L. Wang, A re-interpretation of the STERE/STE observations and its consequences. Astrophys. J. 694, L79-L82 (2009)

W.F. Huebner, J.J. Keady, S.P. Lyon, Solar photo rates for planetary atmospheres and atmospheric pollutants. Astrophys. Space Sci. 195, 1-294 (1992)

M. Juda, R.H. Donnelly, K.T. Hole, A.T. Kenter, R.P. Kraft, S.S. Murray, D.O. Pease, C.R.A. Wilton, M.V. Zombeck, Characteristics of the on-orbit background of the Chandra X-ray observatory high resolution camera. Proc. SPIE 4851, 112-123 (2003) 
R. Kallenbach, M. Hilchenbach, S.V. Chalov, J.A. Le Roux, K. Bamert, On the "injection problem" at the solar wind termination shock. Astron. Astrophys. 439, 1-22 (2005)

L.M. Kistler, F.M. Ipavich, D.C. Hamilton, G. Gloeckler, B. Wilken, G. Kremser, W. Stüdemann, Energy spectra of the major ion species in the ring current during geomagnetic storms. J. Geophys. Res. 94, 3579-3599 (1989)

R. Korde, C. Prince, D. Cunningham, R.E. Vest, E. Gullikson, Present status of radiometric quality silicon photodiodes. Metrologia 40, S145-S149 (2003)

W.F. Krolikowski, W.E. Spicer, Photoemission studies of the noble metals: II. Gold. Phys. Rev. B 1, 478-487 (1970)

D. McComas, S.J. Bame, W.C. Feldman, J.T. Gosling, J.L. Phillips, Solar wind Halo electrons from 1-4 AU. Geophys. Res. Lett. 19, 1291-1294 (1992)

D.J. McComas, P. Valek, J.L. Burch, C.J. Pollock, R.M. Skoug, M.F. Thomsen, Filling and emptying of the plasma sheet: Remote observations with 1-70 keV energetic neutral atoms. Geophys. Res. Lett. 29, 2079 (2002). doi:10.1029/2002GL016153

D. McComas, F. Allegrini, P. Bochsler, M. Bzowski, M. Collier, H. Fahr, H. Fichtner, P. Frisch, H. Funsten, S. Fuselier, G. Gloeckler, M. Gruntman, V. Izmodenov, P. Knappenberger, M. Lee, S. Livi, D. Mitchell, E. Moebius, T. Moore, D. Reisenfeld, E. Roelof, N. Schwadron, M. Wieser, M. Witte, P. Wurz, G. Zank, The interstellar boundary explorer (IBEX). AIP Conf. Proc. 719, 162-181 (2004)

D.J. McComas, F. Allegrini, P. Bochsler, M. Bzowski, M. Collier, H. Fahr, H. Fichtner, P. Frisch, H. Funsten, S. Fuselier, G. Gloeckler, M. Gruntman, V. Izmodenov, P. Knappenberger, M. Lee, S. Livi, D. Mitchell, E. Möbius, T. Moore, S. Pope, D. Reisenfeld, E. Roelof, J. Scherrer, N. Schwadron, R. Tyler, M. Wieser, M. Witte, P. Wurz, G. Zank, IBEX - interstellar boundary explorer. Space Sci. Rev. (2009, this issue)

F.B. McDonald, V.S. Ptuskin, Galactic cosmic rays, in The Century of Space Science (Kluwer, Dordrecht, 2001), pp. 677-697

F. Merkt, R. Signorell, H. Plam, A. Osterwalder, M. Sommavilla, Towards resolving the hyperfine structure in ions by photoelectron spectroscopy. Mol. Phys. 95, 1045-1054 (1998)

D.G. Mitchell, S.E. Jaskulek, C.E. Schlemm, E.P. Keath, R.E. Thompson, B.E. Tossman, J.D. Boldt, J.R. Hayes, G.B. Andrews, N. Paschalidis, D.C. Hamilton, R.A. Lundgren, E.O. Tums, P. Wilson IV, H.D. Voss, D. Prentice, K.C. Hsieh, C.C. Curtis, F.R. Powell, High energy neutral atom (HENA) imager for the IMAGE mission. Space Sci. Rev. 91, 67-112 (2000)

N.P. Paschalidis, E.T. Sarris, S.M. Krimigis, R.W. McEntire, M.D. Levine, I.A. Daglis, G.C. Anagnostopoulos, Energetic ion distributions on both sides of the Earth's magnetopause. J. Geophys. Res. 99, 86878703 (1994)

W.G. Pilipp, H. Miggenrieder, M.D. Montgomery, K.-H. Mühlhäuser, H. Rosenbauer, R. Schwenn, Characteristics of electron velocity distribution functions in the solar wind derived from the HELIOS plasma experiment. J. Geophys. Res. 92, 1075-1092 (1987)

E. Quémerais, R. Lallement, S. Ferron, D. Koutroumpa, J.-L. Bertaux, R. Kyrölä, W. Schmidt, Interplanetary hydrogen absolute ionization rates: Retrieving the solar wind mass flux latitude and cycle dependence with SWAN/SOHO maps. J. Geophys. Res. 111, A09114 (2006). doi:10.1029/2006JA011711

T.R. Sanderson, J.P.G. Henrion, K.-P. Wenzel, R.P. Lin, K.A. Anderson, S. Ashford, C.W. Carlson, D. Curtis, R.F. Ergun, D. Larson, J. McFadden, H. Reme, J.M. Bosqued, J. Coutelier, F. Cotin, N. Lormant, C. d'Uston, G.K. Parks, M.P. McCarthy, R.M. Skoug, R.M. Winglee, WIND observations of energetic ions far upstream of the Earth's bow-shock. Geophys. Res. Lett. 23, 1215-1218 (1996)

J. Steinberg, Private communication. Los Alamos National Laboratory, Los Alamos, NM 87545, USA, 2007

R.G. Tonkyn, R. Wiedmann, E.R. Grant, M.G. White, Rotationally resolved photoionization of $\mathrm{H}_{2} \mathrm{O}$. J. Chem. Phys. 95, 7033-7040 (1991)

J. Vallerga, R. Lallement, M. Lemoine, F. Dalaudier, D. McMullin, Astron. Astrophys. 426, 855-865 (2004)

L. Wang, R.P. Lin, D.E. Larson, J.G. Luhmann, Domination of heliosheath pressure by shock-accelerated pickup ions from observations of neutral atoms. Nature 454, 81-83 (2008)

M. Wieser, P. Wurz, E. Moebius, S.A. Fuselier, E. Hertzberg, D.J. McComas, The ion-optical prototype of the low energy neutral atom sensor of the interstellar boundary explorer mission (IBEX). Rev. Sci. Instr. 78, 124502-1-124502-14 (2007)

D.J. Williams, D.G. Mitchell, L.A. Frank, T.E. Eastman, Three-dimensional magnetosheath plasma ion distributions from $200 \mathrm{eV}$ to $2 \mathrm{MeV}$. J. Geophys. Res. 93, 12783-12794 (1988)

B.E. Wood, V.V. Izmodenov, J.L. Linsky, Y.G. Malama, Ly $\alpha$ Absorption from heliosheath neutrals. Astrophys. J. 657, 609-617 (2007)

P. Wurz, Detection of energetic neutral particles, in The Outer Heliosphere: Beyond the Planets, ed. by K. Scherer, H. Fichtner, E. Marsch (Copernicus Gesellschaft e.V., Katlenburg-Lindau, 2000), pp. 251-288

P. Wurz, J. Scheer, M. Wieser, Particle scattering off surfaces: application in space science. e-J. Surf. Sci. Nanotechnol. 4, 394-400 (2006)

P. Wurz, A. Galli, S. Barabash, A. Grigoriev, Energetic neutral atoms from the heliosheath. Astrophys. J. 683, 248-254 (2008) 